\title{
ON THE BRAUER GROUP OF TORIC VARIETIES
}

\author{
FRANK R. DEMEYER AND TIMOTHY J. FORD
}

\begin{abstract}
We compute the cohomological Brauer group of a normal toric variety whose singular locus has codimension less than or equal to 2 everywhere.
\end{abstract}

Associated to each algebraic variety $X$ is the cohomological Brauer group $\mathrm{B}^{\prime}(X)=\operatorname{tors}\left(\mathrm{H}^{2}\left(X, \mathrm{G}_{m}\right)\right)$ which is the torsion subgroup of the second etale cohomology group of $X$ with coefficients in the sheaf of units. Except in the easiest cases, calculations of this group are scarce. Toric varieties over an algebraically closed field of characteristic 0 provide a nontrivial class of higher dimensional varieties for which calculations of $\mathrm{B}^{\prime}(X)$ can sometimes be made. These calculations are the purpose of this article.

Each toric variety $X$ is determined by a combinatorical object $\Delta$ in real affine space called a fan. Tied into the structure of the fan are arithmetic properties of sublattices of free $\mathbb{Z}$-lattices. Our arguments therefore ultimately reduce questions about $\mathrm{B}^{\prime}(X)$ to calculations with integer matrices.

In $\S 1$ we determine the Brauer group $\mathrm{B}(X)=\mathrm{B}^{\prime}(X)$ of any nonsingular toric variety $X$ (Theorem 1.1 ). This group is a direct sum of finitely many copies of finite cyclic groups and copies of $\mathbb{Q} / \mathbb{Z}$. The algebras generating this group are given explicitly as smash products of cyclic Galois extensions of $X$. In $\S 2$ we consider toric varieties whose singular locus has codimension at most 2 everywhere in $X$. Let $T_{N}$ denote the torus identified with an open subset of $X, \mathrm{~B}^{\prime}\left(T_{N} / X\right)$ the elements in $\mathrm{B}^{\prime}(X)$ split by $T_{N}$, and $\tilde{X}$ a $T_{N}$-invariant desingularization of $X$. In Theorem 2.2 we construct an exact sequence $0 \rightarrow$ $\mathrm{B}^{\prime}\left(T_{N} / X\right) \rightarrow \mathrm{B}^{\prime}(X) \rightarrow \mathrm{B}^{\prime}(\tilde{X}) \rightarrow 0$ which reduces the calculation of $\mathrm{B}^{\prime}(X)$ to the calculation of $\mathrm{B}^{\prime}\left(T_{N} / X\right)$. The hypotheses on $X$ in $\S 2$ imply we can assume the associated fan $\Delta$ contains cones of dimension at most 2 . Corresponding to each cone $\tau_{i}$ of dimension 2 is an irreducible closed subvariety $V_{i}=\operatorname{orb} \tau_{i}$ and an affine neighborhood $U_{\tau_{i}}$ of $V_{i}$ which has a finite cyclic divisor class group $\mathrm{Cl}\left(U_{\tau_{i}}\right)$. If $\Delta$ has 2-dimensional cones $\tau_{1}, \ldots, \tau_{m}$, we construct an exact sequence (Theorem 2.3)

$$
0 \rightarrow \operatorname{Pic}(X) \rightarrow \mathrm{Cl}(X) \rightarrow \bigoplus_{i=1}^{m} \mathrm{Cl}\left(U_{\tau_{i}}\right) \rightarrow \mathrm{B}^{\prime}\left(T_{N} / X\right) \rightarrow 0
$$

Received by the editors May 1, 1990 and, in revised form, October 15, 1990.

1980 Mathematics Subject Classification (1985 Revision). Primary 13A20, 16A16; Secondary 14L32, 14F20.

The second author was supported in part by the NSF under grant DMS-8822944. 
For each prime number $p$ we find a subset $\tau_{1}, \ldots, \tau_{e}$ (after a suitable relabelling) of $\left\{\tau_{1}, \ldots, \tau_{m}\right\}$ such that $\left[\bigoplus_{i=1}^{e} \mathrm{Cl}\left(U_{\tau_{i}}\right)\right]_{p} \cong\left[\mathrm{B}^{\prime}\left(T_{N} / X\right)\right]_{p}$. We calculate the Brauer group of any toric surface (Corollary 2.9). In this case $\mathrm{B}^{\prime}\left(T_{N} / X\right)$ is nontrivial when there is a cycle of divisors on $X$ whose pairwise consecutive intersections are singular points on $X$ whose local rings all have divisor class groups of order divisible by a common prime $p$. An analogous statement holds for $X$ of higher dimension. We employ terminology and notation of [12] for toric varieties and [11] for étale cohomology.

Following the notation terminology of [12] let $r>0$ be an integer and $N=$ $\mathbb{Z}^{r}$ a free abelian group of rank $=r$. Let $\Delta$ be a finite fan on $N_{\mathbb{R}}$ and $X=$ $T_{N} \operatorname{emb}(\Delta)$ the associated toric variety containing the $r$-dimensional torus $T_{N}$ as an open subset defined over the algebraically closed field $k$ of characteristic 0 . Let $N^{\prime}$ be the subgroup of $N$ generated by $\bigcup_{\sigma \in \Delta} \sigma \cap N$. The basis theorem for finitely generated abelian groups gives a basis $n_{1}, \ldots, n_{r}$ of $N$ such that $N^{\prime}=\mathbb{Z} a_{1} n_{1} \oplus \mathbb{Z} a_{2} n_{2} \oplus \cdots \oplus \mathbb{Z} a_{r} n_{r}$ where the $a_{i}$ are nonnegative integers and $a_{i} \mid a_{i+1}$ for $1 \leq i \leq r-1$. Call $\left\{a_{1}, \ldots, a_{r}\right\}$ the set of invariant factors of $\Delta$ (or $\left.X=T_{N} \operatorname{emb}(\Delta)\right)$. Let $\mathrm{B}(X)$ denote the Brauer group of Azumaya algebras on $X$ and $\mathrm{B}^{\prime}(X)$ the torsion subgroup of $\mathrm{H}^{2}\left(X, \mathrm{G}_{m}\right)$ the cohomological Brauer group of $X$. Our principal result of $\S 1$ is

Theorem 1.1. If $X=T_{N} \operatorname{emb}(\Delta)$ is nonsingular and $a_{1}, \ldots, a_{r}$ is the set of invariant factors of $X$, then

$$
\mathbf{B}(X)=\mathbf{B}^{\prime}(X) \cong \bigoplus_{i=1}^{r-1} \operatorname{Hom}\left(\mathbb{Z} / a_{i}, \mathbb{Q} / \mathbb{Z}\right)^{r-i}
$$

We list two special cases of Theorem 1.1.

Corollary 1.2. If $\Delta$ contains a cone $\sigma$ such that $\operatorname{dim} \sigma \geq r-1$, then $\mathrm{B}(X)=(0)$. Proof. Since $X$ is nonsingular, [12, Theorem 1.10] implies there is a basis $n_{1}, \ldots, n_{r}$ of $N$ such that $\mathbb{R}_{\geq 0} n_{1}+\cdots+\mathbb{R}_{\geq 0} n_{r-1} \subseteq \sigma$. Since each $n_{i} \in \sigma \cap N$, all of the invariant factors $a_{i}=1$ for $1 \leq i \leq r-1$. So $\mathrm{B}(X)=(0)$ by Theorem 1.1.

Corollary 1.3. $\mathrm{B}(X)$ is finite if and only if $\operatorname{Rank}_{\mathbb{Z}}\left(N^{\prime}\right) \geq r-1$. In this case

$$
\mathrm{B}(X) \cong \bigoplus_{i=1}^{r-1}\left(\mathbb{Z} / a_{i}\right)^{r-i}
$$

The rest of this section is devoted to a proof of Theorem 1.1. From now on we assume $X=T_{N} \operatorname{emb}(\Delta)$ is nonsingular. Along the way we will obtain explicit information about the Azumaya algebras on $X$ and show the Brauer group $\mathrm{B}(X)$ of Azumaya algebras on $X$ is equal to the cohomological Brauer group $\mathrm{B}^{\prime}(X)=\mathrm{H}^{2}\left(X, \mathrm{G}_{m}\right)$.

Let $\Gamma=\left\{0, \rho_{1}, \ldots, \rho_{n}\right\}$ be the fan on $N_{\mathbb{R}}$ consisting of all cones in $\Delta$ of dimension $\leq 1$ and let $U=T_{N} \operatorname{emb}(\Gamma)$. The open immersion $U \hookrightarrow X$ induces the isomorphisms of the next lemma.

Lemma 1.4. For each positive integer $\nu$,

(a) $\mathrm{H}^{1}(X, \mathbb{Z} / \nu) \cong \mathrm{H}^{1}(U, \mathbb{Z} / \nu)$, 
(b) $\mathrm{H}^{2}(X, \mathbb{Z} / \nu) \cong \mathrm{H}^{2}(U, \mathbb{Z} / \nu)$,

(c) $\mathbf{B}^{\prime}(X) \cong \mathrm{B}^{\prime}(U)$.

Proof. Let $X, U$ be as above and let $Z=X-U$. Then part of the long exact sequence of cohomology with supports [11, Proposition 1.25] is

$$
\begin{gathered}
\mathrm{H}_{Z}^{1}(X, \mathbb{Z} / \nu) \rightarrow \mathrm{H}^{1}(X, \mathbb{Z} / \nu) \rightarrow \mathrm{H}^{1}(U, \mathbb{Z} / \nu) \rightarrow \mathrm{H}_{Z}^{2}(X, \mathbb{Z} / \nu) \\
\rightarrow \mathrm{H}^{2}(X, \mathbb{Z} / \nu) \rightarrow \mathrm{H}^{2}(U, \mathbb{Z} / \nu) \rightarrow \mathrm{H}_{Z}^{3}(X, \mathbb{Z} / \nu)
\end{gathered}
$$

since the codimension of $Z$ in $X$ is $\geq 2$, [11, Lemma 9.1, p. 268] implies $\mathrm{H}_{Z}^{s}(X, \mathbb{Z} / \nu)=(0)$ for $s<4$, which proves (a) and (b) in our context. There is an exact sequence [3, Theorem 1.c]

$$
0 \rightarrow \mathrm{H}^{2}\left(X, \mathrm{G}_{m}\right) \rightarrow \mathrm{H}^{2}\left(U, \mathrm{G}_{m}\right) \rightarrow \mathrm{H}_{Z}^{3}(X, \mu)
$$

and $\mathrm{H}_{Z}^{3}(X, \mu) \cong \stackrel{\lim }{\longrightarrow} \mathrm{H}_{Z}^{3}(X, \mathbb{Z} / \nu)=(0)$, which proves $(\mathrm{c})$.

Notice that $N^{\prime}=\left\langle\bigcup_{\sigma \in \Delta} \sigma \cap N\right\rangle=\left\langle\bigcup_{i=1}^{n} \rho_{i} \cap N\right\rangle$ so as a consequence of Lemma $1.4(\mathrm{c})$ we can assume that $\Delta=\left\{0, \rho_{1}, \ldots, \rho_{n}\right\}$ and $X=U$. We write $\rho_{k}=\mathbb{R}_{\geq 0} \eta_{k}$ where $\eta_{k} \in N$ and $\eta_{k}$ is primitive (the GDC of the coordinates of $\eta_{k}$ is 1$)$. Let $n_{1}, \ldots, n_{r}$ be a basis for $N$ with $N^{\prime}=\mathbb{Z} a_{1} n_{1} \oplus \cdots \oplus \mathbb{Z} a_{r} n_{r}$ and $a_{i} \mid a_{i+1}$ for $1 \leq i \leq r . \quad\left(\left\{a_{1}, \ldots, a_{r}\right\}\right.$ is the set of invariant factors of $\Delta$.) Let $m_{1}, \ldots, m_{r}$ be a dual basis for $M=\operatorname{Hom}(N, \mathbb{Z})$. Then $T_{N}=$ Spec $k[M]$. An element $\sum a_{i} m_{i}$ in $M$ is usually identified with the Laurent monomial $x_{1}^{a_{1}} x_{2}^{a_{2}} \cdots x_{r}^{a_{r}}$ and $k[M]$ with $k\left[x_{1}, x_{1}^{-1}, \ldots, x_{r}, x_{r}^{-1}\right]$. Let $\nu$ be a positive integer and fix a primitive $\nu$ th root of unity $\zeta$. Given units $\alpha, \beta$ in $k\left[x_{1}, x_{1}^{-1}, \ldots, x_{r}, x_{r}^{-1}\right]$, the symbol algebra $(\alpha, \beta)_{\nu}$ is the associative $k$ algebra generated by elements $u, v$ subject to the relations $u^{\nu}=\alpha, v^{\nu}=\beta$; and $u v=\zeta v u$. In what follows, we choose to identify $\left(x_{i}, x_{j}\right)_{\nu}$ as $\left(m_{i}, m_{j}\right)_{\nu}$ and work in $k(M)$. By [10, Theorem 6], ${ }_{\nu} \mathrm{B}\left(T_{N}\right)$ is a free $\mathbb{Z} / \nu$-module with basis given by the set of symbol algebras $\left\{\left(m_{i}, m_{j}\right)_{\nu}\right\}_{1 \leq i<j \leq r}$ for each $\nu \geq 2$. Since $T_{N}$ is an open subset of $X$ and $X$ is nonsingular, $\mathrm{B}(X)$ is a subgroup of $\mathrm{B}\left(T_{N}\right)$ by restriction and our object is to identify this subgroup explicitly.

From [4, Corollary 1.4] there is an exact sequence

$$
0 \rightarrow \mathrm{B}^{\prime}(X) \rightarrow \mathrm{B}\left(T_{N}\right) \stackrel{a}{\longrightarrow} \bigoplus_{i=1}^{n} \mathrm{H}^{1}\left(\operatorname{orb} \rho_{i}, \mathbb{Q} / \mathbb{Z}\right)
$$

where orb $\rho_{i}$ is the $T_{N}$-invariant divisor on $X=T_{N}$ emb $(\Delta)$ corresponding to the face $\rho_{i}$ of $\Delta$. Given a symbol algebra $(\alpha, \beta)_{\nu}$ representing a class in $\mathrm{B}\left(T_{N}\right)$, the ramification map $a$ agrees with the tame symbol (see the discussion following [4, Remark 1.7] and [14, Theorem 8, p. 155]). This means the $k$ th coordinate of $a\left((\alpha, \beta)_{\nu}\right)$, the ramification of $(\alpha, \beta)_{\nu}$ along orb $\rho_{k}$, is identified with a cyclic Galois extension of orb $\rho_{k}$ of degree $\nu$. Over the function field $K$ (orb $\left.\rho_{k}\right)$ this extension is given by adjoining the $\nu$ th root of $\alpha^{v_{k}(\beta)} / \beta^{v_{k}(\alpha)}$ where $v_{k}$ is the valuation on $K(X)$ determined by the prime divisor orb $\rho_{k}$.

From the remarks above, to determine the ramification of an arbitrary algebra $\Lambda$ representing an element in $\mathrm{B}\left(T_{N}\right)$ along orb $\rho_{k}$ it suffices to determine $K$ (orb $\left.\rho_{k}\right)$ and $v_{k}\left(m_{j}\right)$ for each $k, j$. The following lemma is well known. We include its short proof for completeness and to fix notation. 
Lemma 1.5. Let $\eta_{k}$ be the primitive vector in $N \cap \rho_{k}$ and $\langle$,$\rangle the natural$ inner product from $M \times N \rightarrow \mathbb{Z}$.

(a) $K\left(\right.$ orb $\left.\rho_{k}\right)$ is the quotient field of $k\left[\eta_{k}^{\perp}\right]$.

(b) $v_{k}(m)=\left\langle m, \eta_{k}\right\rangle$.

Proof. Since $\eta_{k}$ is primitive there is a primitive $\mu_{k} \in M$ with $\left\langle\mu_{k}, \eta_{k}\right\rangle=1$. Let $\eta_{k}^{\perp}=\left\{m \in M \mid\left\langle m, \eta_{k}\right\rangle=0\right\}$. Then $M=\eta_{k}^{\perp} \oplus \mathbb{Z} \mu_{k}$ since

$$
0 \rightarrow \eta_{k}^{\perp} \rightarrow M \stackrel{\varphi}{\longrightarrow} \mathbb{Z} \rightarrow 0
$$

splits, where $\varphi(m)=\left\langle m, \eta_{k}\right\rangle$. The affine coordinate ring of

$$
U_{\rho_{k}}=T_{N} \operatorname{emb}\left\{0, \rho_{k}\right\}
$$

is $k\left[\eta_{k}^{\perp}, \mu_{k}\right]$. Localizing $X$ along orb $\rho_{k}$ is equivalent to localizing $U_{\rho_{k}}$ along $\operatorname{orb} \rho_{k}$. The prime ideal corresponding to orb $\rho_{k}$ is the principal ideal in $k\left[\eta_{k}^{\perp}, \mu_{k}\right]$ generated by $\mu_{k}$. Hence $\mu_{k}$ is a local parameter along orb $\rho_{k}$. $K\left(\right.$ orb $\left.\rho_{k}\right)$ is the quotient field of $k\left[\eta_{k}^{\perp}, \mu_{k}\right] /\left(\mu_{k}\right)$ giving (a). The valuation $v_{k}$ of any $m \in M$ is the $\mu_{k}$-coordinate when $m$ is written in terms of the decomposition $M=\eta_{k}^{\perp} \oplus \mathbb{Z} \mu_{k}$. Thus $v_{k}(m)=\left\langle m, \eta_{k}\right\rangle$.

Keeping the notation above, define a homomorphism $\operatorname{ram}_{\text {orb } \rho_{k}}:{ }_{\nu} \mathrm{B}\left(T_{N}\right) \rightarrow$ $M / \nu M$ by letting $\operatorname{ram}_{\text {orb } \rho_{k}}\left(m_{i}, m_{j}\right)_{\nu}=\left\langle m_{j}, \eta_{k}\right\rangle m_{i}-\left\langle m_{i}, \eta_{k}\right\rangle m_{j}+\nu M$ be the assignment on the basis for ${ }_{\nu} \mathrm{B}\left(T_{N}\right)$, and extending by $\mathbb{Z} / \nu$-linearity.

Lemma 1.6. $\left(m_{i}, m_{j}\right)_{\nu}$ is unramified along orb $\rho_{k}$ if and only if

$$
\operatorname{ram}_{\text {orb } \rho_{k}}\left(m_{i}, m_{j}\right)_{\nu}=0 .
$$

Proof. The ramification of $\left(m_{i}, m_{j}\right)_{\nu}$ along orb $\rho_{k}$ corresponds to the cyclic extension of the affine coordinate ring $k\left[\eta_{k}^{\perp}\right]$ of orb $\rho_{k}$ obtained by adjoining the $\nu$ th root of $v_{k}\left(m_{j}\right) m_{i}-v_{k}\left(m_{i}\right) m_{j}=\left\langle m_{j}, \eta_{k}\right\rangle m_{i}-\left\langle m_{i}, \eta_{k}\right\rangle m_{j}$. (Note $\left\langle\left\langle m_{j}, \eta_{k}\right\rangle m_{i}-\left\langle m_{i}, \eta_{k}\right\rangle m_{j}, \eta_{k}\right\rangle=0$ so $\left.\left\langle m_{j}, \eta_{k}\right\rangle m_{i}-\left\langle m_{i}, \eta_{k}\right\rangle m_{j} \in \eta_{k}^{\perp}.\right)$ Thus, $\left(m_{i}, m_{j}\right)_{\nu}$ is unramified along orb $\rho_{k}$ if and only if $\left\langle m_{j}, \eta_{k}\right\rangle m_{i}-\left\langle m_{i}, \eta_{k}\right\rangle m_{j}$ is a $\nu$ th power in $k\left[\eta_{k}^{\perp}\right]$ if and only if $\left\langle m_{j}, \eta_{k}\right\rangle m_{i}-\left\langle m_{i}, \eta_{k}\right\rangle m_{j} \in \nu M$ if and only if $\operatorname{ram}_{\text {orb } \rho_{k}}\left(m_{i}, m_{j}\right)_{\nu}=0$.

Let $\Lambda$ be any Azumaya algebra representing a class in ${ }_{\nu} \mathrm{B}\left(T_{N}\right)$. We have seen $\Lambda$ is equivalent to $\prod_{i<j}\left(m_{i}, m_{j}\right)_{\nu}^{e_{i j}}$ where $0 \leq e_{i j}<\nu$. Moreover the class of $\Lambda$ determines and is determined by the integers $e_{i j}$. Associate to the class represented by $\Lambda$ in ${ }_{\nu} \mathrm{B}\left(T_{N}\right)$ the matrix

$$
M_{\Lambda}=\left[\begin{array}{cccccc}
0 & e_{12} & e_{13} & \cdots & & e_{1 r} \\
-e_{12} & 0 & e_{23} & \cdots & & e_{2 r} \\
\cdot & & & & & \cdot \\
\cdot & & & & & \cdot \\
\cdot & & & \cdots & -e_{r-1, r} & 0
\end{array}\right]
$$

Lemma 1.7. (a) The assignment $\Lambda \rightarrow M_{\Lambda}$ induces a monomorphism

$$
\phi:{ }_{\nu} \mathrm{B}\left(T_{N}\right) \rightarrow \operatorname{Hom}_{\mathbb{Z}}(N, M / \nu M) .
$$

(b) $\Lambda$ is unramified along orb $\rho_{k}$ if and only if $M_{\Lambda} \cdot \eta_{k}=0$. 
Proof. (a) The matrix $M_{\Lambda}$ defines the indicated homomorphism $\phi(\Lambda)$ by representing elements in $N$ as column vectors with respect to the basis $n_{1}, \ldots, n_{r}$; the elements in $M$ as column vectors with respect to the dual basis $m_{1}, \ldots, m_{r}$ and following left multiplication by $M_{\Lambda}$ by reduction modulo $\nu M$. Since multiplication of symbols corresponds to addition of exponents modulo $\nu$, it is clear that $\phi$ is a homomorphism. If $\phi(\Lambda)=M_{\Lambda}=0$, then each $e_{i j}=0$, so $\Lambda=0$ in $\mathrm{B}\left(T_{N}\right)$. Thus $\phi$ is a monomorphism.

(b) Write $\eta_{k}=\sum_{i=1}^{r} \eta_{k i} n_{i}$ and let $\Lambda=\prod_{i<j}\left(m_{i}, m_{j}\right)_{\nu}^{e_{i j}}$. Then

$$
\operatorname{ram}_{\mathrm{orb} \rho_{k}}\left(m_{i}, m_{j}\right)_{\nu}=\left\langle m_{j}, \eta_{k}\right\rangle m_{i}-\left\langle m_{i}, \eta_{k}\right\rangle m_{j}=\eta_{k j} m_{i}-\eta_{k i} m_{j} .
$$

Hence

$$
\begin{aligned}
\operatorname{ram}_{\text {orb } \rho_{k}}(\Lambda) & =\operatorname{ram}_{\text {orb } \rho_{k}}\left(\prod_{i<j}\left(m_{i}, m_{j}\right)_{\nu}^{e_{i j}}\right) \\
& =\sum_{i<j} e_{i j} \eta_{k j} m_{i}-e_{i j} m_{j}+\nu M \\
& =\sum_{i=1}^{r} \sum_{j=i+1}^{r} e_{i j} \eta_{k j} m_{i}-\sum_{i=1}^{r} \sum_{j=i+1}^{r} e_{i j} \eta_{k i} m_{j}+\nu M \\
& =\sum_{i=1}^{r} \sum_{j=i+1}^{r} e_{i j} \eta_{k j} m_{i}-\sum_{j=1}^{r} \sum_{i=j+1}^{r} e_{j i} \eta_{k j} m_{i}+\nu M \\
& =M_{\Lambda} \cdot\left[\begin{array}{c}
\eta_{k 1} \\
\vdots \\
\eta_{k r}
\end{array}\right] .
\end{aligned}
$$

As we observed in the proof of Lemma 1.6, $\operatorname{ram}_{\text {orb } \rho_{k}}(\Lambda)=m+\nu M$ for some $m \in \eta_{k}^{\perp}$. The ramification of $\Lambda$ along orb $\rho_{k}$ is the cyclic extension of $k\left[\eta_{k}^{\perp}\right]$ obtained by adjoining the $\nu$ th root of $m$ and this extension is split ( $\Lambda$ is unramified along orb $\rho_{k}$ ) if and only if $m \in \nu \eta_{k}^{\perp}$. Since $\eta_{k}^{\perp}$ is a direct summand of $M, \Lambda$ is unramified along orb $\rho_{k}$ if and only if $m \in \nu M$ if and only if

$$
M_{\Lambda} \cdot\left[\begin{array}{c}
\eta_{k 1} \\
\vdots \\
\eta_{k r}
\end{array}\right]=0
$$

Theorem 1.8. Let $X=T_{N} \operatorname{emb}(\Delta)$ be a nonsingular toric variety and $a_{1}, \ldots, a_{r}$ the set of invariant factors of $X$. Then $\mathrm{B}^{\prime}(X)$ is the subgroup of $\mathrm{B}\left(T_{N}\right)$ represented by algebra classes $\prod_{i<j}\left(m_{i}, m_{j}\right)_{\nu_{i}}^{e_{i j}}$ where $\nu_{i} \mid a_{i}, 1 \leq i \leq r$.

Proof. The exact sequence (1) and Lemma 1.7 imply ${ }_{\nu} \mathrm{B}^{\prime}(X)$ consists of those algebra classes $\Lambda$ in ${ }_{\nu} \mathrm{B}\left(T_{N}\right)$ such that $M_{\Lambda} \cdot \eta_{k}=0$ for the primitive vector $\eta_{k}$ on each 1-dimensional cone $\rho_{k}$ in $\Delta(1 \leq k \leq n)$. If $N^{\prime}$ is the subgroup of $N$ generated by $\bigcup_{\sigma \in \Delta} \sigma \cap N$ then $N^{\prime}$ is generated by $\left\{\eta_{k}\right\}_{k=1}^{n}$ so $\Lambda$ represents a class in ${ }_{\nu} \mathrm{B}^{\prime}(X)$ if and only if $M_{\Lambda}$ vanishes on $N^{\prime}$. For each $\nu>0$ we have 
the commutative diagram with exact rows and columns

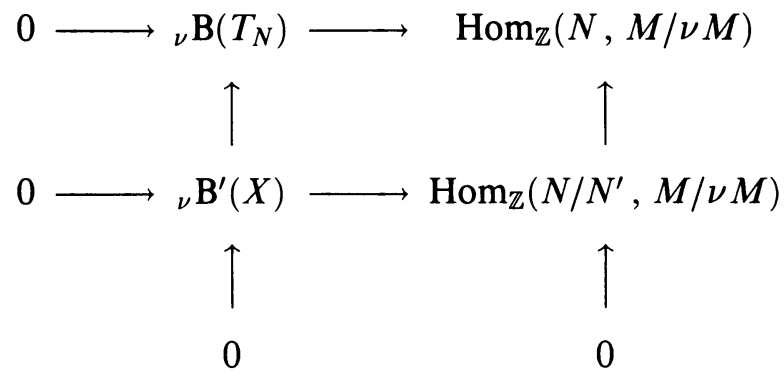

Taking the direct limit over all $\nu \geq 2$ gives a monomorphism

$$
\mathrm{B}^{\prime}(X) \rightarrow \operatorname{Hom}_{\mathbb{Z}}\left(N / N^{\prime}, M \otimes \mathbb{Q} / \mathbb{Z}\right) .
$$

Let $n_{1}, \ldots, n_{r}$ be a basis for $N$ such that $N^{\prime}=\mathbb{Z} a_{1} n_{1} \oplus \cdots \oplus \mathbb{Z} a_{r} n_{r}$ and $a_{i} \mid a_{i+1}$ for $1 \leq i \leq r-1$. That is $a_{1}, \ldots, a_{r}$ is the set of invariant factors for $X$. Then $\operatorname{Hom}_{\mathbb{Z}}\left(N / N^{\prime}, M \otimes \mathbb{Q} / \mathbb{Z}\right) \cong \operatorname{Hom}_{\mathbb{Z}}\left(\bigoplus\left(\mathbb{Z} n_{i} / \mathbb{Z} a_{i} n_{i}\right), M \otimes \mathbb{Q} / \mathbb{Z}\right)$. This means $\mathrm{B}^{\prime}(X)$ is contained in the subgroup of $\mathrm{B}\left(T_{N}\right)$ of algebra classes $\prod_{i<j}\left(m_{i}, m_{j}\right)_{\nu_{i}}^{e_{i j}}$ where $0<\nu_{i}$ and $\nu_{i} \mid a_{i}, 1 \leq i \leq r$. Conversely, if $\nu_{i} \mid a_{i}$ and $\nu_{i} \geq 1$ then the matrix $M_{\Lambda}$ for $\left(m_{i}, m_{j}\right)_{\nu_{i}}$ has a +1 in the $i j$ th entry and a -1 in the jith entry. A typical element in $N^{\prime}$ is $x=\lambda_{1} a_{1} n_{1}+\cdots+\lambda_{r} a_{r} n_{r}$ and $M_{\Lambda} \cdot x=\lambda_{j} a_{j} m_{i}-\lambda_{i} a_{i} m_{j} \in a_{i} M$. Thus $\left(m_{i}, m_{j}\right)_{\nu_{i}}$ represents an element in $\mathbf{B}^{\prime}(X)$. So $\mathbf{B}^{\prime}(X)=\left\{\prod_{i<j}\left(m_{i}, m_{j}\right)_{\nu_{i}}^{e_{i j}} \mid 0<\nu_{i}\right.$ and $\left.\nu_{i} \mid a_{i}\right\}$.

Now it follows that $\mathbf{B}^{\prime}(X) \cong \bigoplus_{i=1}^{r-1} \operatorname{Hom}\left(\mathbb{Z} / a_{i}, \mathbb{Q} / \mathbb{Z}\right)^{r-i}$. To complete the proof of Theorem 1.1 it suffices to show $\mathrm{B}(X)=\mathrm{B}^{\prime}(X)$. It suffices to find an Azumaya algebra $\Lambda$ on $X$ such that $K(X) \otimes \Lambda$ is equivalent to $\left(m_{i}, m_{j}\right)_{\nu_{i}}$ for each $\nu_{i} \mid a_{i}$.

Lemma 1.9. Let $X$ be as in Theorem 1.8 and let $N^{\prime}=\left\langle\bigcup_{\sigma \in \Delta} \sigma \cap N\right\rangle$. Let $\nu \geq 2$ and let $M_{\nu}=\left\{m \in M \mid\left\langle m, n^{\prime}\right\rangle \equiv 0(\bmod \nu)\right.$ for all $\left.n^{\prime} \in N^{\prime}\right\}$. If $\left(\nu, a_{i}\right)$ is the greatest common divisor of $\nu$ and $a_{i}$, then

$$
\mathbf{H}^{1}(X, \mathbb{Z} / \nu) \cong M_{\nu} / \nu M \cong \bigoplus_{i=1}^{r} \mathbb{Z} /\left(\nu, a_{i}\right)
$$

Proof. Restriction induces an embedding $\mathrm{H}^{1}(X, \mathbb{Z} / \nu) \rightarrow \mathrm{H}^{1}\left(T_{N}, \mathbb{Z} / \nu\right)$. The correspondence which assigns to each element $m \in M$ the cyclic extension of $T_{N}$ obtained by adjoining the $\nu$ th root of $m$ induces an isomorphism $M / \nu M \cong$ $\mathrm{H}^{1}\left(T_{N}, \mathbb{Z} / \nu\right)$. An element $m+\nu M$ corresponds to an element of $\mathrm{H}^{1}(X, \mathbb{Z} / \nu)$ if and only if $K(X)\left(m^{1 / \nu}\right)$ is unramified along orb $\rho_{k}$ for $1 \leq k \leq n$ if and only if the restriction of $m$ to orb $\rho_{k}$ is a unit in the coordinate ring $k\left[\eta_{k}^{\perp}, \mu_{k}\right]$ of orb $\rho_{k}$ if and only if $v_{k}(m) \equiv 0(\bmod \nu)$ if and only if $\left\langle m, \eta_{k}\right\rangle \equiv 0(\bmod \nu)$ where unexplained notation is as in Lemma 1.5. Thus

$$
\begin{aligned}
\mathrm{H}^{1}(X, \mathbb{Z} / \nu) & =\left\{m \in M \mid\left\langle m, \eta_{k}\right\rangle \equiv 0(\bmod \nu)(1 \leq k \leq n)\right\}+\nu M \\
& =\left\{m \in M \mid\left\langle m, n^{\prime}\right\rangle \equiv 0(\bmod \nu) \text { for all } n^{\prime} \in N^{\prime}\right\}+\nu M \\
& =M_{\nu} / \nu M .
\end{aligned}
$$

But $N^{\prime}=\mathbb{Z} a_{1} n_{1} \oplus \cdots \oplus \mathbb{Z} a_{r} n_{r}$ so it is easy to check that

$$
M_{\nu} / \nu M \cong \bigoplus_{i=1}^{r} \mathbb{Z}\left(\frac{\nu}{\left(a_{i}, \nu\right)} m_{i}\right) / \mathbb{Z}\left(\nu m_{i}\right) \cong \bigoplus_{i=1}^{r} \mathbb{Z} /\left(a_{i}, \nu\right) \text {. }
$$


Lemma 1.10. If $X$ is as in Theorem 1.8, then $\mathrm{B}(X)=\mathrm{B}^{\prime}(X)$.

Proof. It suffices to show each $\left(m_{i}, m_{j}\right)_{\nu_{i}}$ is in the image of the cup product map $\mathrm{H}^{1}\left(X, \mathbb{Z} / \nu_{i}\right) \times \mathrm{H}^{1}\left(X, \mu_{\nu_{i}}\right) \rightarrow \mathrm{B}(X)$ when $\nu_{i} \mid a_{i}$, since cup products correspond to taking smash products of cyclic Galois extensions and thus are Azumaya algebras (e.g. [6]).

If $a_{j}=0$, both $m_{i}, m_{j} \in M_{\nu_{i}}$ since $\nu_{i} /\left(a_{i}, \nu_{i}\right)=\nu_{i} / \nu_{i}=1=\nu_{i} /\left(0, \nu_{i}\right)$. If $a_{j} \neq 0$, then $\left(m_{i}, m_{j}\right)_{\nu_{i}} \sim\left(m_{i}, m_{j}\right)_{a_{j}}^{a_{j} / \nu_{i}} \sim\left(\left(a_{j} / \nu_{i}\right) m_{i}, m_{j}\right)_{a_{j}}$. But $a_{j} /\left(a_{i}, a_{j}\right)$ $=a_{j} / a_{i}$ which divides $a_{j} / \nu_{i}$ since $\nu_{i} \mid a_{i}$. Thus $\left(a_{j} / \nu_{i}\right) m_{i}$ and $m_{j}$ are both in $M_{a_{j}}$ and $\left(m_{i}, m_{j}\right)_{\nu_{i}}$ is equivalent to an algebra in the image of the cup product $\operatorname{map} \mathrm{H}^{1}\left(X, \mathbb{Z} / a_{j}\right) \times \mathrm{H}^{1}\left(X, \mu_{a_{j}}\right) \rightarrow{ }_{a_{j}} \mathrm{~B}(X)$. So $\mathrm{B}(X)=\mathrm{B}^{\prime}(X)$.

As a result of observations made so far, we can show the following proposition.

Proposition 1.11. Let $\prod_{i<j}\left(m_{i}, m_{j}\right)_{\nu}^{e_{i j}}$ represent a class in $\mathrm{B}\left(T_{N}\right)$ of order $\nu$. Let $M_{\Lambda}$ be the matrix transformation in $\operatorname{Hom}(N, M / \nu M)$ defined in Lemma 1.7 and let $t$ be the rank of $\operatorname{kernel}\left(M_{\Lambda}\right)$. Then there exists a direct summand $P$ of $M$ with $\operatorname{rank}(P)=r-t$ and an Azumaya algebra $L$ over $k[P]$ with $\Lambda \cong k[M] \otimes_{k[P]} L$. No direct summand of $M$ of smaller rank has this property. Proof. Find a basis $n_{1}, \ldots, n_{r}$ of $N$ such that $\operatorname{ker}\left(M_{\Lambda}\right)=\mathbb{Z} b_{1} n_{1} \oplus \mathbb{Z} b_{2} n_{2} \oplus$ $\cdots \oplus \mathbb{Z} b_{r} n_{r}$ and $b_{i} \mid b_{i+1}$ for $1 \leq i \leq r-1$. Since $\operatorname{ker}\left(M_{\Lambda}\right)$ has rank $t, b_{t} \neq 0$ and $b_{t+i}=0$ for $i \geq 1$.

Let $P$ be a direct summand of $M$ and assume $\Lambda$ is obtained by extending an algebra over $k[P]$. Let $m_{1}^{\prime}, \ldots, m_{s}^{\prime}$ be a basis for $P$ and extend this basis to a basis for $M$. We can assume $\Lambda=\prod_{i<j \leq s}\left(m_{i}^{\prime}, m_{j}^{\prime}\right)_{\nu}^{\boldsymbol{e}_{i j}^{\prime}}$. If $n_{1}^{\prime}, \ldots, n_{r}^{\prime}$ is the dual basis to $m_{1}^{\prime}, \ldots, m_{r}^{\prime}$, then the matrix of the transformation $M_{\Lambda}$ with respect to this new basis pair has a kernel which contains a direct summand of $N$ of rank $r-s$. Therefore $t \geq r-s$ so $s \geq r-t$. Now let $m_{1}, \ldots, m_{r}$ be a dual basis for $M$ with respect to $n_{1}, \ldots, n_{r}$. The matrix $M_{\Lambda}$ with respect to this new basis is

$$
M_{\Lambda}=\left[\begin{array}{c|cccc}
0 & \multicolumn{4}{c}{0} \\
\hline & 0 & e_{t+1, t} & \cdots & e_{t+1, r} \\
0 & -e_{t+1, t} & 0 & \cdots & \\
& \vdots & & & \vdots \\
& -e_{t+1, r} & & \cdots & 0
\end{array}\right] .
$$

So $\Lambda$ is defined on the torus $k\left[m_{t+1}, \ldots, m_{r},-m_{t+1}, \ldots,-m_{r}\right]$ and we can take $P=\left\langle m_{t+1}, \ldots, m_{r}\right\rangle$. The rank of $P$ is $r-t$.

In this section we continue to let $\Delta$ be a finite fan on $N_{\mathbb{R}}$ and $X=T_{N} \operatorname{emb}(\Delta)$ the associated toric variety containing the $r$-dimensional torus $T_{N}$ as an open subset. Assume $\Delta$ consists of cones of dimension $\leq 2$ and let $\Delta(2)=\left\{\tau_{1}, \ldots\right.$, $\left.\tau_{m}\right\}$. Let $U_{i}=U_{\tau_{i}}, V_{i}=V\left(\tau_{i}\right)=\operatorname{orb}\left(\tau_{i}\right)$ and let $V=V_{1} \cup \cdots \cup V_{m}$. Then $X-V=T_{N} \operatorname{emb}(\Delta-\Delta(2))$ is nonsingular. In this situation our first lemma gives information about the étale cohomology groups of the affine open subsets $U_{i}$ of $X$. 
Lemma 2.1. (a) For each $i$ and each $p \geq 0$, we have a short exact sequence

$$
0 \rightarrow \mathrm{H}^{p}\left(U_{i}, \mathrm{G}_{m}\right) \rightarrow \mathrm{H}^{p}\left(U_{i}-V_{i}, \mathrm{G}_{m}\right) \rightarrow \mathrm{H}_{V_{i}}^{p+1}\left(U_{i}, \mathrm{G}_{m}\right) \rightarrow 0 .
$$

(b) $\mathrm{H}^{p}\left(U_{i}, \mathrm{G}_{m}\right) \cong \mathrm{H}^{p}\left(T_{r-2}, \mathrm{G}_{m}\right)$ where $T_{r-2}$ is a torus of dimension $r-2$.

Proof. First we check that $\mathrm{H}^{p}\left(U_{i}, \mathrm{G}_{m}\right)$ is torsion for $p \geq 2$. For notational simplicity we suppress the subscript $i$ from $\tau_{i}, U_{i}$, and $V_{i}$. Now $\tau$ is a twodimensional cone in $N_{\mathbb{R}}$. Let $\bar{\tau}$ be $\tau$ viewed as a two-dimensional cone in $\mathbb{R} \tau$. Then $U=U_{\bar{\tau}} \times T_{r-2}$ where $T_{r-2}$ is an $(r-2)$-dimensional torus. Let $R$ be the affine coordinate ring of $U_{\bar{\tau}}$, and $R^{h}$ the henselization of $R$ at the maximal ideal $m$ corresponding to the closed point orb $\bar{\tau}$. Let $R\left[X, X^{-1}\right]$ denote the affine coordinate ring of $U$ and let $U^{h}=\operatorname{Spec} R^{h}\left[X, X^{-1}\right]$. Let $V^{h}=V \times U^{h}$. Then $V^{h}$ is the closed set corresponding to $I=m R^{h}\left[X, X^{-1}\right]$. The completion of $R^{h}\left[X, X^{-1}\right]$ in the $I$-adic topology is $\widehat{R}\left[X, X^{-1}\right]$ where $\widehat{R}$ is the $m$-adic completion of $R$. By [13, p. 127], we see that $\left(R^{h}\left[X, X^{-1}\right], I\right)$ is a Hensel pair. By [5, p. 35] $\mathrm{Cl}\left(R^{h}\left[X, X^{-1}\right]\right)$ embeds into $\mathrm{Cl}\left(\widehat{R}\left[X, X^{-1}\right]\right)$. Since the singularity of $U$ is given by a finite cyclic group action $[12, \mathrm{p} .30]$, it is well known that $\mathrm{Cl}\left(\widehat{R}\left[X, X^{-1}\right]\right)=\mathrm{Cl}(\widehat{R})$ is also finite cyclic [2, Satz 2.11]. Thus $\mathrm{Cl}\left(U^{h}\right)$ is finite. The long exact sequences for the pairs $V \subseteq U$ and $V^{h} \subseteq U^{h}$ give the commutative diagram

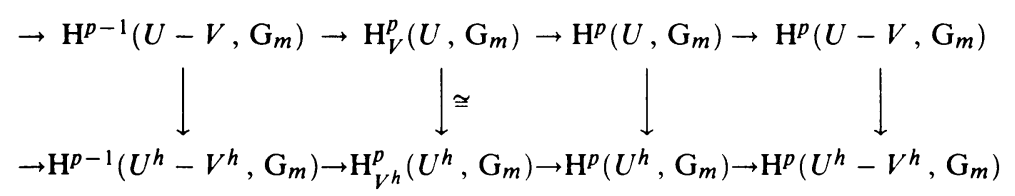

with exact rows. By excision $\mathrm{H}_{V}^{p}\left(U, \mathrm{G}_{m}\right) \cong \mathrm{H}_{V^{h}}^{p}\left(U^{h}, \mathrm{G}_{m}\right)$ [11, p. 92]. By [15] $\mathrm{H}^{p}\left(U^{h}, \mathrm{G}_{m}\right) \cong \mathrm{H}^{p}\left(V^{h}, \mathrm{G}_{m}\right)=\mathrm{H}^{p}\left((\operatorname{orb} \bar{\tau}) \times T_{r-2}, \mathrm{G}_{m}\right)$ which is torsion for $p \geq 2$ since $T_{r-2}$ is smooth [7, p. 71]. Again by [7, p. 71] $\mathrm{H}^{p}\left(U^{h}-V^{h}, \mathrm{G}_{m}\right)$ and $\mathrm{H}^{p}\left(U-V, \mathrm{G}_{m}\right)$ are torsion for $p \geq 2$. But $\mathrm{H}^{1}\left(U^{h}-V^{h}, \mathrm{G}_{m}\right)=\operatorname{Pic}\left(U^{h}-V^{h}\right)=$ $\mathrm{Cl}\left(U^{h}-V^{h}\right)=\mathrm{Cl}\left(U^{h}\right)$ is torsion. It now follows that $\mathrm{H}^{p}\left(U, \mathrm{G}_{m}\right)$ is torsion for $p \geq 2$.

The natural map $U \times A^{1} \rightarrow U$ and Kummer theory induce the commutative diagram

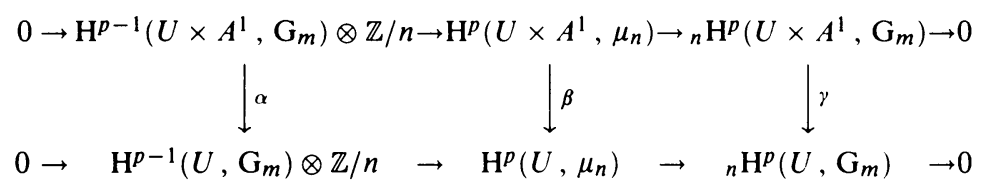

for all $p \geq 2$ and $n \geq 2$. By [11, p. 240] $\beta$ is an isomorphism for $p \geq 2$. Since $\operatorname{Pic} U=0=\operatorname{Pic}\left(U \times A^{1}\right), \alpha$ is an isomorphism for $p=2$. Therefore, $\gamma$ is an isomorphism for $p=2$ and all $n \geq 2$. Taking the inductive limit over all $n$, we have $\mathrm{H}^{2}\left(U \times A^{1}, \mathrm{G}_{m}\right) \cong \mathrm{H}^{2}\left(U, \mathrm{G}_{m}\right)$. By induction on $p$ we see that $\mathrm{H}^{p}\left(U \times A^{1}, \mathrm{G}_{m}\right) \cong \mathrm{H}^{p}\left(U, \mathrm{G}_{m}\right)$ for all $p \geq 2$.

We can give the coordinate ring $k\left[\mathscr{S}_{\tau}\right]$ of $U$ a grading by the nonnegative integers such that the degree $=0$ subring is the coordinate ring of $T_{r-2}$. Since $\mathrm{H}^{p}\left(U \times A^{1}, \mathrm{G}_{m}\right)=\mathrm{H}^{p}\left(U, \mathrm{G}_{m}\right),\left[8\right.$, Theorem 1.1] implies $\mathrm{H}^{p}\left(U, \mathrm{G}_{m}\right) \cong$ 
$\mathrm{H}^{p}\left(T_{r-2}, \mathrm{G}_{m}\right)$, which proves (b). We have a commutative diagram

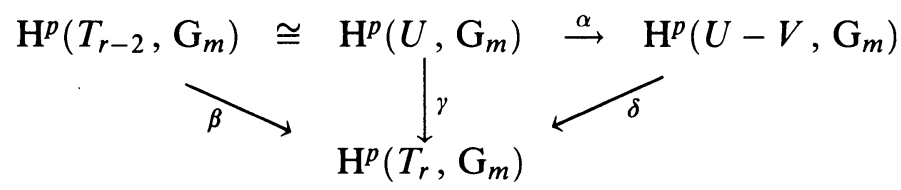

where the maps $\beta, \gamma, \delta$ are induced from restriction. Since $\beta$ is injective, $\alpha$ is injective and Lemma 2.1 now follows.

Theorem 2.2. Let $\Delta$ be a fan which consists of cones of dimension $\leq 2$. Let $\Delta^{\prime}$ be a nonsingular fan obtained from $\Delta$ by subdividing the two-dimensional faces of $\Delta$ and let $\widetilde{X}=T_{N} \mathrm{emb}\left(\Delta^{\prime}\right)$. Then the sequence $0 \rightarrow \mathrm{B}^{\prime}\left(T_{N} / X\right) \rightarrow \mathrm{B}^{\prime}(X) \rightarrow$ $\mathrm{B}^{\prime}(\widetilde{X}) \rightarrow 0$ (with natural maps) is exact.

Proof. Let $\pi: \widetilde{X} \rightarrow X$ be the desingularization resulting from the subdivision $\Delta^{\prime}$ of $\Delta\left[12\right.$, Corollary 1.18] and let $\widetilde{U}_{i}=\pi^{-1}\left(U_{i}\right)$. From the long exact sequence of cohomology with supports, and the observation that $V$ is a disjoint union of closed sets $V_{i}$ (see pp. 92-93 of [11]) we have a commutative diagram with exact rows

$$
\begin{aligned}
& 0 \rightarrow \mathbf{B}^{\prime}\left(T_{N} / X\right) \rightarrow \mathbf{B}^{\prime}(X) \rightarrow \mathbf{B}^{\prime}(X-V) \longrightarrow \bigoplus_{i=1}^{m} \mathbf{H}_{V_{i}}^{3}\left(U_{i}, \mathrm{G}_{m}\right) \\
& \downarrow \alpha \quad \downarrow=\quad \downarrow \\
& 0 \quad \rightarrow \mathbf{B}^{\prime}(\tilde{X}) \rightarrow \mathbf{B}^{\prime}\left(\tilde{X}-\pi^{-1}(V)\right) \rightarrow \bigoplus_{i=1}^{m} \mathrm{H}_{\pi^{-1}\left(V_{i}\right)}^{3}\left(\widetilde{U}_{i}, \mathrm{G}_{m}\right)
\end{aligned}
$$

The second row is exact since $\tilde{X}$ is nonsingular. First check $\beta$ is injective. For each $i$, Lemma 2.1 yields the commutative diagram with exact rows

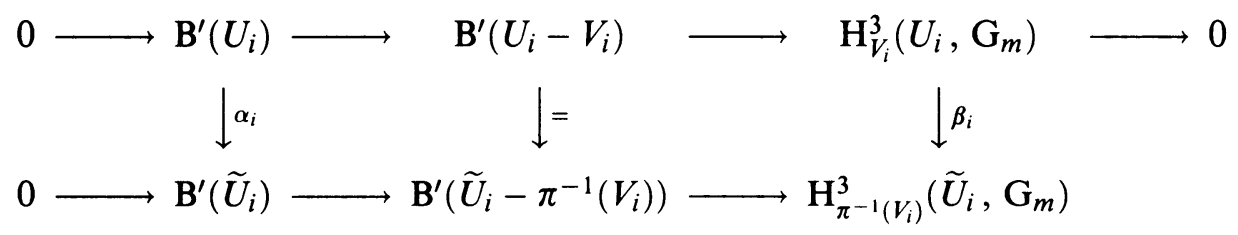

Here $\mathrm{B}^{\prime}\left(U_{i}\right)=\mathrm{B}^{\prime}\left(U_{\bar{\tau}_{i}} \times T_{r-2}\right)=\mathrm{B}\left(T_{r-2}\right)$ by Lemma 2.1 and $\mathrm{B}^{\prime}\left(U_{i}-V_{i}\right)=$ $\mathrm{H}^{2}\left(U_{i}-V_{i}, \mathrm{G}_{m}\right)$ since $U_{i}-V_{i}$ is nonsingular. If $\Delta^{\prime}\left(\tau_{i}\right)$ is the fan whose cones are the cones of $\Delta^{\prime}$ contained in $\tau_{i}$, then $\Delta^{\prime}\left(\tau_{i}\right)$ is a nonsingular fan whose one dimensional faces lie in a plane. The invariants for $\widetilde{U}_{i}=T_{N} \operatorname{emb}\left(\Delta^{\prime}\left(\tau_{i}\right)\right)$ are $\{1,1,0, \ldots, 0\}$ and Theorem 1.1 implies $\mathbf{B}^{\prime}\left(\widetilde{U}_{i}\right)=\mathbf{B}^{\prime}\left(\widetilde{U}_{\bar{\tau}_{i}} \times T_{r-2}\right)=\mathbf{B}\left(T_{r-2}\right)$ so $\alpha_{i}$ is an isomorphism. Since $\operatorname{ker} \beta_{i}=\operatorname{coker} \alpha_{i}, \beta_{i}$ is injective so $\beta$ is injective. But $\operatorname{ker} \beta=\operatorname{coker} \alpha$, so $\alpha$ is an epimorphism and the theorem follows.

As a result of Theorem 2.2 and our analysis of the Brauer groups of nonsingular toric varieties in $\S 1$, we are left with the study of $\mathrm{B}^{\prime}\left(T_{N} / X\right)$.

Theorem 2.3. Let $\Delta$ be a fan which consists of cones of dimension $\leq 2$. Let $\Delta(2)=\left\{\tau_{1}, \ldots, \tau_{m}\right\}$. Let $X=T_{N} \mathrm{emb}(\Delta)$ and let $U_{i}=U_{\tau_{i}}$ be the open subsets 
of $X$ associated to the $\tau_{i}$. Then there is an exact sequence

$$
0 \rightarrow \operatorname{Pic}(X) \rightarrow \mathrm{Cl}(X) \rightarrow \bigoplus_{i=1}^{m} \mathrm{Cl}\left(U_{i}\right) \rightarrow \mathrm{B}^{\prime}\left(T_{N} / X\right) \rightarrow 0
$$

Proof. Let $V_{i}=V\left(\tau_{i}\right)=\operatorname{orb}\left(\tau_{i}\right)$ and let $V=V_{1} \cup \cdots \cup V_{m}$. From the long exact sequence of cohomology with supports in the closed set $V$ we have (since $V$ is the disjoint union of the closed sets $V_{i}$ )

$$
\begin{aligned}
\cdots & \rightarrow \mathrm{H}^{1}\left(X, \mathrm{G}_{m}\right) \rightarrow \mathrm{H}^{1}\left(X-V, \mathrm{G}_{m}\right) \rightarrow \bigoplus_{i=1}^{m} \mathrm{H}_{V_{i}}^{2}\left(X, \mathrm{G}_{m}\right) \\
& \rightarrow \mathrm{H}^{2}\left(X, \mathrm{G}_{m}\right) \rightarrow \mathrm{H}^{2}\left(X-V, \mathrm{G}_{m}\right) \rightarrow \bigoplus_{i=1}^{m} \mathrm{H}_{V_{i}}^{3}\left(X, \mathrm{G}_{m}\right) \rightarrow \cdots
\end{aligned}
$$

Since $V$ has codimension 2 in $X$, and $X-V$ is nonsingular, $\mathrm{H}^{1}\left(X-V, \mathrm{G}_{m}\right)=$ $\operatorname{Pic}(X-V)=\mathrm{Cl}(X-V)=\mathrm{Cl}(X)$. Since $U_{i}$ is an open neighborhood of $V_{i}$, $\mathrm{H}_{V_{i}}^{p}\left(X, \mathrm{G}_{m}\right)=\mathrm{H}_{V_{i}}^{p}\left(U_{i}, \mathrm{G}_{m}\right)$ for all $p \geq 0$ [11, p. 93]. From Lemma 2.1 with $p=1$ we get the exact sequences

$$
0 \rightarrow \operatorname{Pic} U_{i} \rightarrow \mathrm{Cl} U_{i} \rightarrow \mathrm{H}_{V_{i}}^{2}\left(U_{i}, \mathrm{G}_{m}\right) \rightarrow 0 \quad(1 \leq i \leq m) .
$$

Lemma 2.1(b) gives $\operatorname{Pic} U_{i}=\operatorname{Pic} T_{r-2}=0$ so $\mathrm{Cl}\left(U_{i}\right)=\mathrm{H}_{V_{i}}^{2}\left(U_{i}, \mathrm{G}_{m}\right)$. Since $\tau_{i}$ is simplicial, $\mathrm{Cl}\left(U_{i}\right)=\operatorname{Pic}\left(U_{i}-V_{i}\right)$ is torsion [12, Proposition 2.1]. Since $X-V$ is nonsingular, $\mathrm{B}^{\prime}(X-V)=\mathrm{H}^{2}\left(X-V, \mathrm{G}_{m}\right)$ and $\mathrm{B}^{\prime}(X-V) \rightarrow \mathrm{B}\left(T_{N}\right)$ is injective. But $\operatorname{Pic} X \rightarrow \mathrm{Cl}(X)$ is injective [5]. With these identifications (1) reduces to the sequence of the theorem.

Corollary 2.4. In the context of Theorem 2.3, if $\operatorname{rank}_{\mathbb{Z}}(N)=r \leq 3$ and $m \geq 1$, then

$$
0 \rightarrow \operatorname{Pic}(X) \rightarrow \mathrm{Cl}(X) \rightarrow \bigoplus_{i=1}^{m} \mathrm{Cl}\left(U_{i}\right) \rightarrow \mathrm{B}^{\prime}(X) \rightarrow 0
$$

is exact.

Proof. We need to check $\mathbf{B}^{\prime}(X)=\mathbf{B}^{\prime}\left(T_{N} / X\right) . \mathrm{H}^{2}\left(U_{i}, \mathrm{G}_{m}\right)=\mathrm{H}^{2}\left(T_{r-2}, \mathrm{G}_{m}\right)=$ 0 for $r \leq 3$ (Lemma 2.1 and [10]). From Lemma 2.1(a) we have (since $U_{i}-V_{i}$ is nonsingular) $\mathrm{B}^{\prime}\left(U_{i}-V_{i}\right)=\mathrm{H}^{2}\left(U_{i}-V_{i}, \mathrm{G}_{m}\right)=\mathrm{H}_{V_{i}}^{3}\left(U_{i}, \mathrm{G}_{m}\right)=\mathrm{H}_{V_{i}}^{3}\left(X, \mathrm{G}_{m}\right)$ so (1) becomes

$$
0 \rightarrow \operatorname{Pic} X \rightarrow \mathrm{Cl}(X) \rightarrow \bigoplus_{i=1}^{m} \mathrm{Cl}\left(U_{i}\right) \rightarrow \mathrm{B}^{\prime}(X) \rightarrow \mathrm{B}^{\prime}(X-V) \rightarrow \bigoplus_{i=1}^{m} \mathrm{~B}^{\prime}\left(U_{i}-V_{i}\right)
$$

Since $X-V$ is nonsingular, restriction induces a monomorphism $\mathrm{B}^{\prime}(X-V) \rightarrow$ $\mathrm{B}^{\prime}\left(U_{i}-V_{i}\right)$ for each $i$ and the corollary follows.

The object of the rest of this section is to give an algorithm for finding for each prime $p$ a subset $\tau_{1}, \ldots, \tau_{e}$ of the two-dimensional faces of $\Delta$ such that $\left[\bigoplus_{i=1}^{e} \mathrm{Cl}\left(U_{\tau_{i}}\right)\right]_{p} \cong\left[\mathrm{B}^{\prime}\left(T_{N} / X\right)\right]_{p}$. (For $G$ a finite abelian group, $G_{p}$ is the Sylow $p$-subgroup.) In particular, the exact sequence of Theorem 2.3 is splitexact. To the fan $\Delta$ we associated a bipartite graph $\Gamma$. The vertex set of $\Gamma$ is $\Delta(1) \cup \Delta(2)=\left\{\rho_{1}, \ldots, \rho_{n}\right\} \cup\left\{\tau_{1}, \ldots, \tau_{m}\right\}$ and there is an edge in $\Gamma$ connecting $\rho_{j}$ and $\tau_{j}$ if and only if $\rho_{j}$ is a face of $\tau_{i}$. If $Y$ is the $T_{N}$-invariant divisor $X-T_{N}$ on $X$, then $\Gamma$ is the graph associated to $Y$ in the sense of [4]. A 
cycle $Z$ in $\Gamma$ (i.e., $Z$ is homeomorphic to the unit circle) determines a finite set $\tau_{1}, \ldots, \tau_{l}$ of two-dimensional cones and $\rho_{1}, \ldots, \rho_{l}$ of one-dimensional faces of $\Delta$ configured as follows:

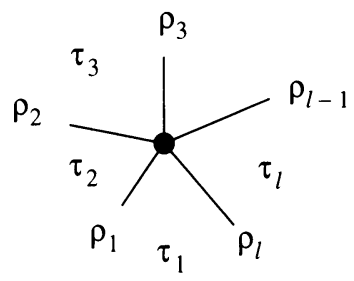

If $\Delta_{Z}$ is the subfan of $\Delta$ consisting of the cones $\left\{0, \rho_{1}, \ldots, \rho_{l}, \tau_{1}, \ldots, \tau_{l}\right\}$, we will show that the cohomological Brauer group of $T_{N} \operatorname{emb}\left(\Delta_{Z}\right)$ is cyclic of order the greatest common divisor of $\left\{\left|\mathrm{Cl} U_{\tau_{i}}\right|\right\}_{i=1}^{l}$. Of course, there may be many such cycles in $\Gamma$ and the last step in the analysis is to choose for each prime $p$ a list of cycles $\left\{Z_{i}\right\}_{i=1}^{e}$ and for each $Z_{i}$ a face $\tau_{i}$ such that $\left[\bigoplus_{i=1}^{e} \mathrm{Cl}\left(U_{\tau_{i}}\right)\right]_{p} \cong\left[\mathrm{B}^{\prime}\left(T_{N} / X\right)\right]_{p}$.

We adopt the following notation: for each two-dimensional cone $\tau_{i}$ in $\Delta$ $(1 \leq i \leq m)$ let $\rho_{i 1}$ and $\rho_{i 2}$ be the one-dimensional faces of $\tau_{i}$ so $\tau_{i}=$ $\rho_{i 1}+\rho_{i 2}$. We have observed $\mathrm{Cl}(X)=\mathrm{Cl}(X-V)=\operatorname{Pic}(X-V)$ and $\mathrm{Cl}\left(U_{i}\right)=$ $\mathrm{Cl}\left(U_{i}-V_{i}\right)=\operatorname{Pic}\left(U_{i}-V_{i}\right)$. Now we want to present $\operatorname{Pic}(X-V)$ and $\operatorname{Pic}\left(U_{i}-V_{i}\right)$ in terms of support functions on the fan $\Delta-\left\{\tau_{1}, \ldots, \tau_{m}\right\}$. If we let $\rho_{1}, \ldots, \rho_{n}$ be the one-dimensional cones in $\Delta$, then we can identify the support functions on $\Delta-\left\{\tau_{1}, \ldots, \tau_{m}\right\}$ with the direct sum of copies of $\mathbb{Z}$ indexed by the $\rho_{i}$. If $\Delta_{i}=\left\{0, \rho_{i 1}, \rho_{i 2}\right\}$, then $U_{i}-V_{i}=T_{N} \operatorname{emb}\left(\Delta_{i}\right)$. It follows from [12, Corollary 2.5] that the sequences

$$
\begin{aligned}
& M \rightarrow \bigoplus_{i=1}^{n} \mathbb{Z} \rho_{i} \rightarrow \mathrm{Cl}(X) \rightarrow 0, \\
& M \stackrel{\beta}{\rightarrow} \mathbb{Z} \rho_{i 1} \oplus \mathbb{Z} \rho_{i 2} \rightarrow \mathrm{Cl}\left(U_{i}\right) \rightarrow 0
\end{aligned}
$$

are exact. Combining these sequences with the exact sequence of Theorem 2.3 gives a commutative diagram with exact rows and columns

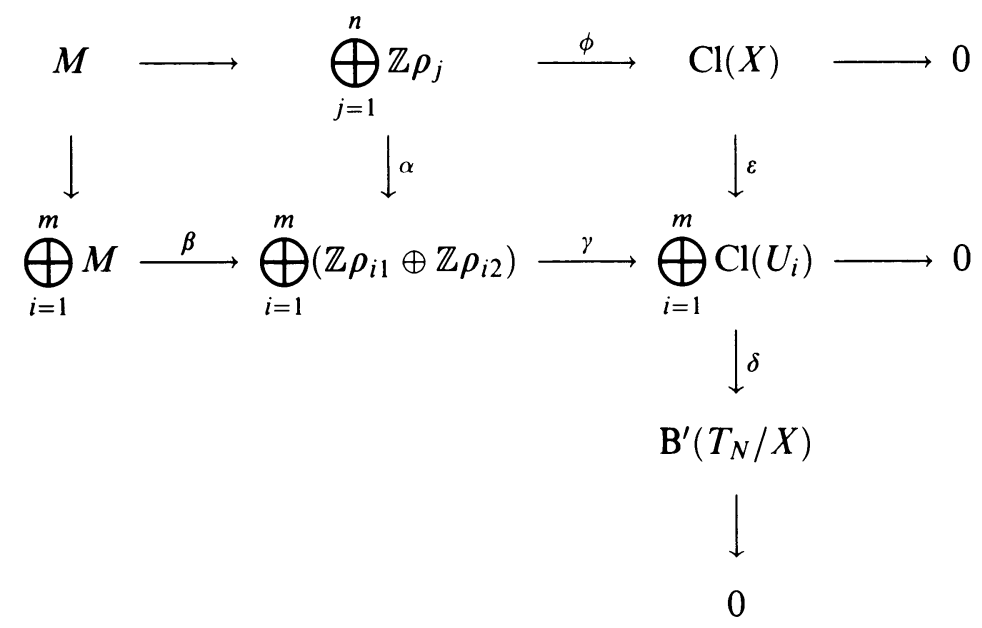


It is routine to check that $\operatorname{im} \alpha+\operatorname{im} \beta=\operatorname{ker} \delta \gamma$. As a result we have a fundamental exact sequence which we exploit for the remainder of this section: (in this sequence $\psi=\delta \gamma$ ),

$$
\left(\bigoplus_{j=1}^{n} \mathbb{Z} \rho_{j}\right) \oplus\left(\bigoplus_{i=1}^{m} M\right) \stackrel{\alpha+\beta}{\longrightarrow} \bigoplus_{i=1}^{m}\left(\mathbb{Z} \rho_{i 1} \oplus \mathbb{Z} \rho_{i 2}\right) \stackrel{\psi}{\longrightarrow} \mathrm{B}^{\prime}\left(T_{N} / X\right) \rightarrow 0
$$

Let $\Gamma$ be the graph associated to $\Delta$. Observe that $\Gamma$ has $2 m$ edges since each $\tau_{i}$ has exactly two one-dimensional faces $\rho_{i 1}$ and $\rho_{i 2}$. The free abelian group $\bigoplus_{i=1}^{m}\left(\mathbb{Z} \rho_{i 1} \oplus \mathbb{Z} \rho_{i 2}\right)$ is called the edge space of $\Gamma$. If we write $\Gamma$ as a union of its connected components $\Gamma_{i}$ we get a corresponding decomposition of $\Delta$ into subfans $\Delta_{i}$ with $\Delta_{i} \cap \Delta_{j}=\{0\}$ whenever $i \neq j$. The decomposition of $\Delta$ gives an open cover of $X$ where the elements in the open over are $T_{N} \operatorname{emb}\left(\Delta_{i}\right)=X_{i}$ and $X_{i} \cap X_{j}=T_{N}$ whenever $i \neq j$. With this notation we can prove

Proposition 2.5. The natural map $\mathbf{B}^{\prime}(X) \rightarrow \bigoplus_{i} \mathbf{B}^{\prime}\left(X_{i}\right)$ induces an isomorphism $\mathbf{B}^{\prime}\left(T_{N} / X\right) \cong \bigoplus_{i} \mathbf{B}^{\prime}\left(T_{N} / X_{i}\right)$.

Proof. Assume $\Delta=\Delta_{1} \cup \Delta_{2}$ where $\Delta_{1}$ and $\Delta_{2}$ are fans with $\Delta_{1} \cap \Delta_{2}=\{0\}$. It is sufficient to prove $\mathrm{B}^{\prime}\left(T_{N} / X\right) \cong \mathrm{B}^{\prime}\left(T_{N} / X_{1}\right) \oplus \mathrm{B}^{\prime}\left(T_{N} / X_{2}\right)$ where $X_{i}=T_{N} \operatorname{emb}\left(\Delta_{i}\right)$ $(i=1,2)$. Let $\Delta_{1}(1)=\left\{\rho_{1}, \ldots, \rho_{n_{1}}\right\}$ and $\Delta_{1}(2)=\left\{\tau_{1}, \ldots, \tau_{m_{1}}\right\}$ and $\Delta_{2}(1)=\left\{r_{1}, \ldots, r_{n_{2}}\right\}$ and $\Delta_{2}(2)=\left\{t_{1}, \ldots, t_{m_{2}}\right\}$. Also let $\tau_{i}=\rho_{i 1}+\rho_{i 2}$ and $t_{j}=r_{j 1}+r_{j 2}$ where $\rho_{i k} \in \Delta_{1}(1)$ and $r_{j l} \in \Delta_{2}(1)$. With respect to this decomposition the exact sequence (3) decomposes as

$$
\begin{gathered}
{\left[\bigoplus_{j=1}^{n_{1}} \mathbb{Z} \rho_{j} \oplus \bigoplus_{i=1}^{m_{1}} M\right] \oplus\left[\bigoplus_{j=1}^{n_{2}} \mathbb{Z} r_{j} \oplus \bigoplus_{i=1}^{m_{2}} M\right] \stackrel{\left(\alpha_{1}+\beta_{1}\right) \oplus\left(\alpha_{2}+\beta_{2}\right)}{\longrightarrow}} \\
{\left[\bigoplus_{i=1}^{m_{1}}\left(\mathbb{Z} \rho_{i 1} \oplus \mathbb{Z} \rho_{i 2}\right)\right] \oplus\left[\bigoplus_{i=1}^{m_{2}}\left(\mathbb{Z} r_{i 1} \oplus \mathbb{Z} r_{i 2}\right)\right] \rightarrow \mathrm{B}^{\prime}\left(T_{N} / X\right) \rightarrow 0}
\end{gathered}
$$

But coker $\left(\alpha_{1}+\beta_{1}\right) \oplus\left(\alpha_{2}+\beta_{2}\right)=\mathrm{B}^{\prime}\left(T_{N} / X_{1}\right) \oplus \mathrm{B}^{\prime}\left(T_{N} / X_{2}\right)$ by (3) so $\mathrm{B}^{\prime}\left(T_{N} / X\right)=$ $\mathbf{B}^{\prime}\left(T_{N} / X_{1}\right) \oplus \mathbf{B}^{\prime}\left(T_{N} / X_{2}\right)$.

Notice in Proposition 2.5 that if $X_{i}$ corresponds to a connected component of $\Gamma$ containing no two-dimensional faces $\tau_{i}$ as vertices, then $X_{i}=T_{N} \operatorname{emb}\{0, \rho\}$ for some one-dimensional cone $\rho$ in $\Delta$. In this case $X_{i}$ is nonsingular and $\mathrm{B}^{\prime}\left(T_{N} / X_{i}\right)=0$. Thus, as a result of Proposition 2.5 we can assume $\Gamma$ is connected and at least one vertex of $\Gamma$ is a two-dimensional cone in $\Delta$.

We now determine a matrix representation for the map $\alpha+\beta$ in (3). Let $\tau=\rho_{1}+\rho_{2} \in \Delta(2)$ and consider the map

$$
M \stackrel{\beta}{\longrightarrow} \mathbb{Z} \rho_{1} \oplus \mathbb{Z} \rho_{2} \rightarrow \mathrm{Cl}\left(U_{\tau}\right) \rightarrow 0
$$

as in (2). Pick a basis $n_{1}, \ldots, n_{r}$ for $N$ and a dual basis $m_{1}, \ldots, m_{r}$ for $M$. Let $\eta_{i}$ be a primitive element in $N$ with $\rho_{i}=\mathbb{R}_{\geq 0} \eta_{i}$. The matrix of $\beta$ with respect to the basis pair $\left\{m_{1}, \ldots, m_{r}\right\},\left\{\rho_{1}, \rho_{2}\right\}$ is the $2 \times r$ matrix whose $i, j$ th entry is $\left\langle m_{j}, \eta_{i}\right\rangle$. But $\left\langle m_{j}, \eta_{i}\right\rangle$ is the $j$ th coordinate of $\eta_{i}$ so we can write this matrix as $\left(\begin{array}{l}\eta_{1} \\ \eta_{2}\end{array}\right)$ where we think of $\eta_{i}$ as a row vector. Therefore the $\operatorname{map} \beta$ in (3)

$$
\bigoplus_{i=1}^{m} M \stackrel{\beta}{\longrightarrow} \bigoplus_{i=1}^{m}\left(\mathbb{Z} \rho_{i 1} \oplus \mathbb{Z} \rho_{i 2}\right)
$$


has a matrix representation which is a direct sum of $2 \times r$ matrices $\left(\begin{array}{l}\eta_{i 1} \\ \eta_{i 2}\end{array}\right)$ where $\eta_{i 1}$ and $\eta_{i 2}$ are the primitive generators of $\rho_{i 1}$ and $\rho_{i 2}$ expressed with respect to the basis $\left\{n_{1}, \ldots, n_{r}\right\}$. To determine the matrix for the homomorphism

$$
\bigoplus_{j=1}^{n} \mathbb{Z} \rho_{j} \stackrel{\alpha}{\longrightarrow} \bigoplus_{i=1}^{m}\left(\mathbb{Z} \rho_{i 1} \oplus \mathbb{Z} \rho_{i 2}\right)
$$

given in (3) we observe the $j$ th column of this matrix is $\alpha\left(\rho_{j}\right)$. Thus the $j$ th column has a 1 in the row determined by $\rho_{i k}$ if $\rho_{j}=\rho_{i k}$. Otherwise this entry is 0 . The matrix of the homomorphism $\alpha+\beta$ of (3) is then

$$
Q=\left[\alpha\left(\rho_{1}\right) \cdots \alpha\left(\rho_{n}\right) \mid \begin{array}{lll}
\left(\begin{array}{l}
\eta_{11} \\
\eta_{12}
\end{array}\right) & & \\
& \ddots & \\
& & \left(\begin{array}{l}
\eta_{m 1} \\
\eta_{m 2}
\end{array}\right)
\end{array}\right]
$$

Note $Q$ is an integral matrix with $2 m$ rows and $n+r m$ columns, and we can identify $\operatorname{im}(\alpha+\beta)$ with the column space of $Q$. Since $\mathrm{B}^{\prime}\left(T_{N} / X\right) \cong \operatorname{coker}(\alpha+\beta)$ from (3), calculating $\mathrm{B}^{\prime}\left(T_{N} / X\right)$ is reduced to determining the column space of $Q$. Our first observation is a straightforward calculation:

$$
Q \cdot\left[\begin{array}{c}
\eta_{1} \\
\vdots \\
\eta_{n} \\
-I_{r} \\
\vdots \\
-I_{r}
\end{array}\right]=(0)
$$

where $I_{r}$ is the $r \times r$ identity matrix. Thus the last $r$ columns of $Q$ containing $\left(\begin{array}{l}\eta_{m 1} \\ \eta_{m 2}\end{array}\right)$ are linear combinations of the preceding columns. We now assume $\Gamma$ is connected, and let $T$ be a spanning tree for $\Gamma$. We observe that in $\Gamma$ each vertex $\tau_{i}$ is joined by edges $\tau_{i-} \rho_{i 1}, \tau_{i-} \rho_{i 2}$ to vertices $\rho_{i 1}, \rho_{i 2}$ so there are $2 m$ edges in $\Gamma$. Since $\Gamma$ is connected, there are $n+m-1$ edges in $T$ [1].

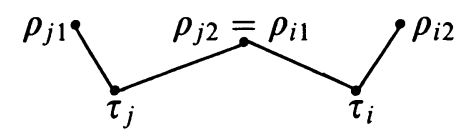

Thus, if $c_{1}, \ldots, c_{e}$ denote the edges of $\Gamma$ that are not in $T$, then $e=m-n+1$ and for each $i$ at least one of $\tau_{i-} \rho_{i 1}, \tau_{i-} \rho_{i 2}$ is in $T$. By reindexing we can assume $c_{1}=\tau_{1-} \rho_{12}, \ldots, c_{e}=\tau_{e}-\rho_{e 2}$. We identify $c_{j}$ with the basis vector $\rho_{j 2}$ in the edge space $\bigoplus_{i=1}^{m}\left(\mathbb{Z} \rho_{i 1} \oplus \mathbb{Z} \rho_{i 2}\right)$. For $1 \leq i \leq m$ let $n_{i 1}$ be a primitive vector in $N$ with $\mathbb{R}_{\geq 0} n_{i 1}=\rho_{i 1}$ and choose $n_{i 2}$ in $N$ with $\left\{n_{i 1}, n_{i 2}\right\}$ a basis for $\mathbb{R} \tau_{i} \cap N$. We can extend $\left\{n_{i 1}, n_{i 2}\right\}$ to a basis $\left\{n_{i 1}, n_{i 2}, \ldots, n_{i r}\right\}$ for $N$. With respect to this basis we can write $\eta_{i 1}=n_{i 1}, \eta_{i 2}=a_{i} n_{i 1}+b_{i} n_{i 2}$ where the $\eta_{i j}$ are as in $Q$. With respect to these basis choices for $N$ and corresponding dual basis choices for $M$, and after deleting columns consisting of zeros, the matrix $Q$ for $\alpha+\beta$ becomes 


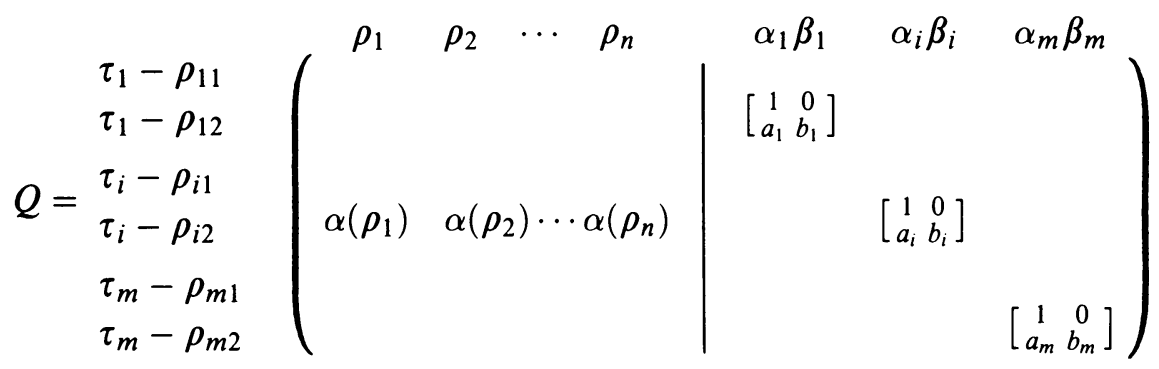

where the first $n$ columns span $\operatorname{im} \alpha$ and the last $2 m$ columns span $\operatorname{im} \beta$. We checked in (4) above that the last 2 columns labeled $\alpha_{m}$ and $\beta_{m}$ are linear combinations of the preceding $n+2(m-1)$ columns. It follows from (2) that $\left|b_{i}\right|=\left|\mathrm{Cl}\left(U_{\tau_{i}}\right)\right|=\left|\mathrm{Cl} U_{i}\right|$. The columns $\beta_{1}, \ldots, \beta_{e}$ are $b_{1} c_{1}, \ldots, b_{e} c_{e}$.

Theorem 2.6. Let $\Delta$ be a fan on $N_{\mathbb{R}}$ and let $X=T_{N} \operatorname{emb}(\Delta)$. Assume all the cones in $\Delta$ have dimension $\leq 2$. Assume the two-dimensional faces $\tau_{1}, \ldots, \tau_{m}$ and one-dimensional faces $\rho_{1}, \ldots, \rho_{n}$ of $\Delta$ can be ordered so that $\tau_{i} \cap \tau_{i+1}=\rho_{i}$ $(1 \leq i \leq m-1)$ and $\tau_{m} \cap \tau_{1}=\rho_{m}$. Let $b_{i}$ be the order of $\operatorname{Cl}\left(U_{\tau_{i}}\right)$. Then $\mathbf{B}^{\prime}\left(T_{N} / X\right)$ is cyclic of order $\operatorname{gcd}\left\{b_{1}, \ldots, b_{m}\right\}$.

Proof. Using Proposition 2.5 and the hypotheses, we can assume that the graph $\Gamma$ is connected and consists of one cycle as shown:

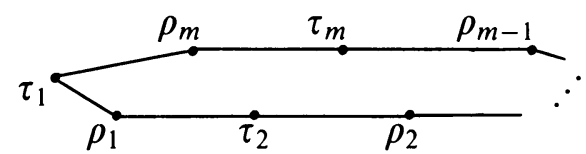

We take the spanning tree $T$ for $\Gamma$ to be the graph obtained from $\Gamma$ by deleting the edge $c_{1}=\tau_{1-} \rho_{m}$. Let $C$ be the matrix whose only column is $c_{1}$ and form the augmented matrix $[Q \mid C]$ :

$$
\begin{aligned}
& \begin{array}{lllllllllllllllllllllll}
\rho_{1} & \rho_{2} & \rho_{3} & \ldots & \rho_{m-2} & \rho_{m-1} & \rho_{m} & \alpha_{1} & \beta_{1} & \alpha_{2} & \beta_{2} & \ldots & \alpha_{m-1} & \beta_{m-1} & \alpha_{m} & \beta_{m} & c_{1}
\end{array}
\end{aligned}
$$

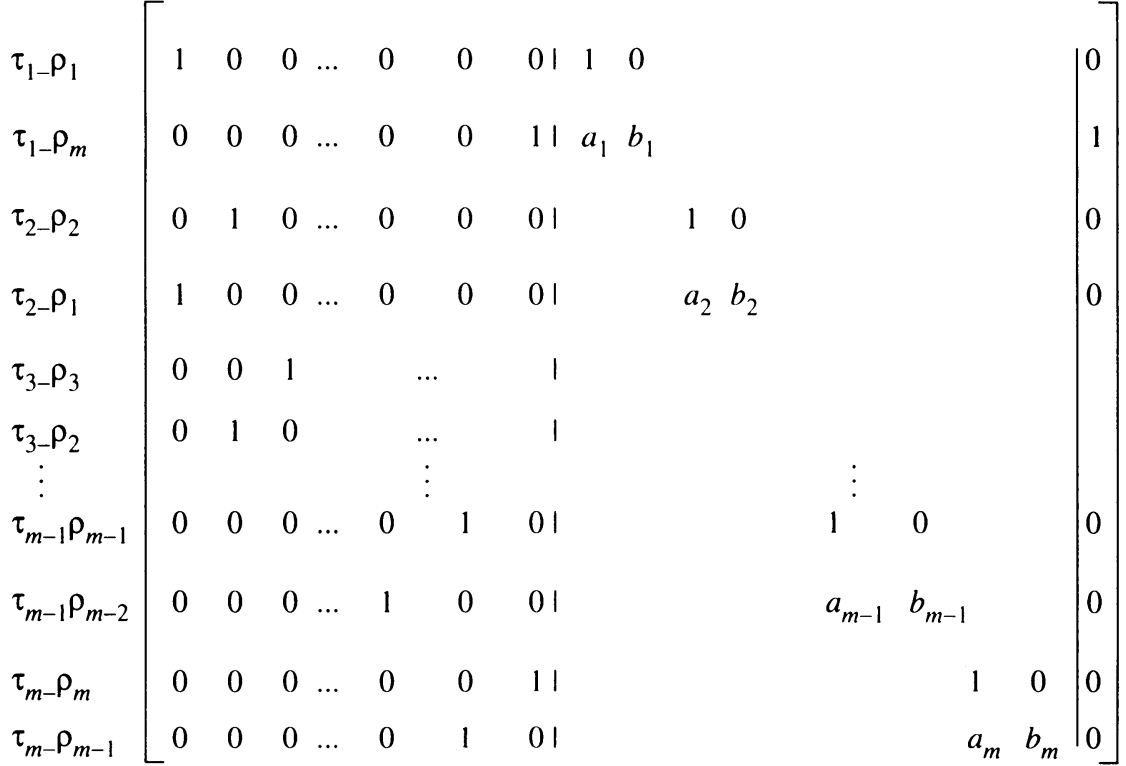


Here $c_{1}$ corresponds to the edge $\tau_{1-} \rho_{m}$ and $\bigoplus_{i=1}^{m}\left(\mathbb{Z} \rho_{i} \oplus \mathbb{Z} \rho_{i-1}\right)$ is the edge space of $\Gamma$ where $\rho_{0}=\rho_{m}$. We observed that $\mathrm{B}^{\prime}\left(T_{N} / X\right)$ is the quotient of the edge space by the column space of $Q$. Let $[B \mid C]$ be the matrix whose columns are the columns labeled $\rho_{1}, \ldots, \rho_{m}, \alpha_{1}, \alpha_{2}, \ldots, \alpha_{m-1}, c_{1}$. We check that the columns of $[B \mid C]$ form a basis for the edge space by using column operations to reduce to a permutation matrix. Use column $c_{1}$ to eliminate $a_{1}$ from column $\alpha_{1}$ and the 1 in the entry with row index $\tau_{1-} \rho_{m}$ and column index $\rho_{m}$. Then use the 1 in the new column $\alpha_{1}$ to eliminate the 1 in the entry with row index $\tau_{1-} \rho_{1}$ and column index $\rho_{1}$. Use the new column $\rho_{1}$ to eliminate the $a_{2}$ in column $\alpha_{2}$. Continue inductively, eliminating $a_{3}, \ldots, a_{m-2}$ from columns indexed $\alpha_{3}, \ldots, \alpha_{m-2}$ and the ones in the entries with row index $\tau_{i-} \rho_{i}$ and column index $\rho_{i}$, for $2 \leq i \leq m-2$. At the last step use the remaining 1 in column $\rho_{m-2}$ to eliminate $a_{m-1}$ in column $\alpha_{m-1}$. Use 1 in the new column $\alpha_{m-1}$ to eliminate the 1 in the entry in row $\tau_{m-1}-\rho_{m-1}$, column $\rho_{m-1}$. The result is a matrix whose $\mathbb{Z}$-rank is $2 m$, which shows that $c_{1}$ generates the quotient of the edge space $\bigoplus_{i=1}^{m}\left(\mathbb{Z} \rho_{i} \oplus \mathbb{Z} \rho_{i-1}\right) \quad\left(\rho_{0}=\rho_{m}\right)$ by the column space of $Q$. Recall that the last two columns of $Q$ are a linear combination of the preceeding ones. Thus to calculate this quotient we simply project each of the columns $\beta_{1}, \ldots, \beta_{m}$ on $\mathbb{Z} c_{1}$. These projections follow the recursive pattern:

$$
\begin{array}{lr}
\frac{\text { Column vector }}{\beta_{1}=b_{1} c_{1}} & \text { Projection on } \mathbb{Z} c_{1} \\
\beta_{2}=b_{2}\left(\rho_{1}-\left(\alpha_{1}-a_{1} c_{1}\right)\right) & b_{1} c_{1} \\
\beta_{3}=b_{3}\left(\rho_{2}-\left(\alpha_{2}-a_{2}\left(\rho_{1}-\left(\alpha_{1}-a_{1} c_{1}\right)\right)\right)\right) & b_{3} a_{2} a_{1} c_{1} \\
\vdots & \vdots \\
\beta_{m} & b_{m-1} a_{m-1} \cdots a_{2} a_{1} c_{1}
\end{array}
$$

The subgroup generated by the projections of the columns $\beta_{i}$ on $\mathbb{Z} c_{1}$ is the subgroup generated by $d c_{1}$ where $d=\operatorname{gcd}\left\{b_{1}, b_{2} a_{1}, \ldots, b_{m} a_{m-1} \cdots a_{2} a_{1}\right\}$. Since $\operatorname{gcd}\left(a_{i}, b_{i}\right)=1$ for $1 \leq i \leq m$, we see $d=\operatorname{gcd}\left\{b_{1}, \ldots, b_{m}\right\}$. But $\left|b_{i}\right|$ is the order of $\mathrm{Cl}\left(U_{\tau_{i}}\right)$, so the theorem follows.

To extend Theorem 2.6 it is necessary to introduce some additional notation. Suppose the graph $\Gamma$ we have associated to the fan $\Delta$ is connected and let $T$ be a spanning tree for $\Gamma$. Since each vertex labeled by a two-dimensional face $\tau_{i}$ is connected by exactly two edges to vertices $\rho_{i 1}$ and $\rho_{i 2}$ corresponding to the one-dimensional faces of $\tau_{i}$ in $\Delta$, each $\tau_{i}$ is a vertex in $T$. If $\Delta(2)=$ $\left\{\tau_{1}, \ldots, \tau_{m}\right\}$, designate $\tau_{m}$ as the root node for $T$. Let $C$ be the matrix whose columns are $c_{1}, \ldots, c_{e}$ and let $[Q \mid C]$ be the augmented matrix similar to that used in the proof of Theorem 2.6. Then $\tau_{1}, \ldots, \tau_{e}$ are leaf nodes of $T$ and $c_{i}=\tau_{i-} \rho_{i 2}$ for $1 \leq i \leq e$. For $e<i<m$ relabel $\rho_{i 1}$ and $\rho_{i 2}$ if necessary so the edge $\tau_{i-} \rho_{i 1}$ is closer to the root node $\tau_{m}$ than the edge $\tau_{i-} \rho_{i 2}$. In our previous analysis this amounts to permuting the basis of the edge space $\bigoplus_{i=1}^{m}\left(\mathbb{Z} \rho_{i 1} \oplus \mathbb{Z} \rho_{i 2}\right)$. This does not affect the columns labeled $\alpha_{1}, \beta_{1}, \ldots, \alpha_{e}, \beta_{e}$ in $Q$. Let $[B \mid C]$ be the matrix obtained from $[Q \mid C]$ by deleting from $Q$ the columns labeled $\beta_{1}, \ldots, \beta_{m-1}, \beta_{m}, \alpha_{m}$. We note that the column space of $B$ depends on the choice of $\tau_{m}$.

Lemma 2.7. The columns of $[B \mid C]$ form a basis for $\bigoplus_{i=1}^{m}\left(\mathbb{Z} \rho_{i 1} \oplus \mathbb{Z} \rho_{i 2}\right)$, the edge space, for any choice of root node $\tau_{m}$. 
Proof. If $\Gamma$ is a tree, then $T=\Gamma$ and $e=0$. In this case we need to show that the columns of $B$ span the edge space. Each leaf node of $\Gamma$ must be $\rho_{i 2}$ for some $i$ since each $\tau_{i}$ is incident to two edges in $\Gamma=T$ and $\rho_{i 1}$ is closer to the root node $\tau_{m}$ than $\rho_{i 2}$. We call the pair $\left(\tau_{i}, \rho_{i 2}\right)$ a leaf node pair. If $i=m$, then it is possible for $\rho_{i 1}$ to be a leaf node. This is the only exception and will be treated in the basis step for our induction below. Assume $\left(\tau_{i}, \rho_{i 2}\right)$ is a leaf node pair and $i \neq m$. In $\Delta, \rho_{i 2}$ is a face of exactly one two-dimensional cone $\tau_{i}$. Thus the column indexed by $\rho_{i 2}$ in $B$ has exactly one nonzero entry which is a 1 in the row indexed $\tau_{i-} \rho_{i 2}$ as indicated below.

$$
\tau_{i}-\rho_{i l}\left[\begin{array}{ccc}
\rho_{i l} & \rho_{i 2} & \alpha_{i} \\
1 & 0 & 1 \\
0 & 1 & a_{i}
\end{array}\right]
$$

Use the column indexed $\rho_{i 2}$ to eliminate $a_{i}$ in the column $\alpha_{i}$ by an elementary column operation, then use the new column $\alpha_{i}$ to eliminate the 1 in the $\tau_{i-} \rho_{i 1}$ entry of column $\rho_{i 1}$. After these two steps are performed, we say we have pruned the leaf node pair from the tree $\Gamma=T$. The two columns indexed $\rho_{i 2}$ and $\alpha_{i}$ are now elementary basis vectors in our basis for the edge space and appear in no further column operations. After the columns indexed $\rho_{i 2}$ and $\alpha_{i}$ are deleted, the remaining matrix is the matrix we would associate to the fan $\Delta^{\prime}$ obtained from $\Delta$ be deleting the cones $\rho_{i 2}$ and $\tau_{i}$. Apply this leaf pruning algorithm iteratively to reduce to the case where $\Gamma$ is the tree $\rho_{m 1-} \tau_{m-} \rho_{m 2}$. The matrix $B$ for this tree is $\left[\begin{array}{ll}1 & 0 \\ 0 & 1\end{array}\right]$. Thus our algorithm reduces the original matrix $B$, using elementary column operations, to a permutation matrix.

If $\Gamma$ is not a tree, let $\tau_{1-} \rho_{12}, \ldots, \tau_{e-} \rho_{e 2}$ be the edges of $\Gamma$ which are not in $T$. Fix $i, 1 \leq i \leq e$. Since $\tau_{i-} \rho_{i 1}$ is in $T$, it follows that $\tau_{i}$ is a leaf node of $T$. We can use the column indexed $c_{i}$ and elementary column operations to eliminate the entry $a_{i}$ from the column indexed $\alpha_{i}$ and the entry 1 in the column indexed $\rho_{i 2}$ and row indexed $\tau_{i-} \rho_{i 2}$ in the matrix $[B \mid C]$. Use the new column $\alpha_{i}$ to eliminate the 1 in the $\tau_{i-} \rho_{i 1}$ entry of column $\rho_{i 1}$. Repeat this step for $i=1, \ldots, e$. Observe that the $2 e \times 2 e$ submatrix of $[B \mid C]$ whose columns are indexed $\alpha_{i}(1 \leq i \leq e), c_{i}(1 \leq i \leq e)$ and whose rows are indexed $\tau_{i-} \rho_{i j} \quad(1 \leq i \leq e$ and $1 \leq j \leq 2)$ has rank $2 e$. If we delete this submatrix from $[B \mid C]$ (which corresponds to deleting $\tau_{1}, \ldots, \tau_{e}$ from the fan $\Delta$ ), the resulting matrix is the one we would associate to the fan $\Delta^{\prime}=\Delta-\left\{\tau_{1}, \ldots, \tau_{e}\right\}$. The graph of $\Delta^{\prime}$ is a tree so the result follows from the first part of the proof.

Corollary 2.8. Let $\Delta$ be a fan on $\mathbb{R}^{r}$ whose cones all have dimension $\leq 2$. If the graph $\Gamma$ associated to $\Delta$ is a disjoint union of trees and $X=T_{N} \operatorname{emb}(\Delta)$, then $\mathrm{B}^{\prime}\left(T_{N} / X\right)=0$.

Proof. By Proposition 2.5 we can assume $\Gamma$ is connected, so the hypotheses imply $\Gamma$ is a tree. By Lemma 2.7 the columns of $B$ span the edge space of $\Gamma$. But the column space of $B$ is contained in $\operatorname{im}(\alpha+\beta)$ in (3) $\mathrm{B}^{\prime}\left(T_{N} / X\right)=0$.

Corollary 2.9. Let $\Delta$ be a fan on $\mathbb{R}^{2}$ and $X=T_{N} \operatorname{emb}(\Delta)$ the associated toric surface.

(a) If $\Delta=\{0\}$, then $\mathrm{B}(X) \cong \mathbb{Q} / \mathbb{Z}$.

(b) If $\Delta \neq\{0\}$ and $|\Delta| \neq \mathbb{R}^{2}$ (i.e., $X$ is not complete), then $\mathrm{B}(X)=0$.

(c) If $|\Delta|=\mathbb{R}^{2}$ (i.e., $X$ is complete), $\Delta(1)=\left\{\rho_{1}, \ldots, \rho_{n}\right\}$ and $N^{\prime}=$ $\left\langle\rho_{1} \cap N, \ldots, \rho_{n} \cap N\right\rangle$, then $\mathrm{B}(X) \cong N / N^{\prime}$. 
Proof. Every toric surface is a normal projective surface [12] so by [9, Corollary 9] $\mathrm{B}(X)=\mathrm{B}^{\prime}(X)$. If $\Delta=\{0\}$, then $X=T_{N}$ is nonsingular and since $r=2$, $\mathrm{B}(X)=\mathbb{Q} / \mathbb{Z}[10]$. If $\Delta \neq\{0\}$ and $|\Delta| \neq \mathbb{R}^{2}$, then the graph $\Gamma$ associated to $\Delta$ is a disjoint union of trees. By Proposition 2.5 we assume $\Gamma$ is a tree and contains at least one two-dimensional cone $\tau$. By Corollary 2.8 and Corollary 2.4, $\mathrm{B}(X)=0$. If $|\Delta|=\mathbb{R}^{2}$, then $\Gamma$ is a cycle. Corollary 2.4 implies $\mathrm{B}(X)=$ $\mathrm{B}\left(T_{N} / X\right)$. If $\Delta(2)=\left\{\tau_{1}, \ldots, \tau_{m}\right\}$, then Theorem 2.6 implies $\mathrm{B}(X)$ is cyclic of order $\operatorname{gcd}\left\{\left|\mathrm{Cl}\left(U_{\tau_{i}}\right)\right|\right\}_{i=1}^{m}$. Let $\rho_{1}, \ldots, \rho_{m}$ be the one-dimensional cones in $\Delta$ with $\tau_{i}=\rho_{i}+\rho_{i+1} \quad(1 \leq i \leq m)$ where $\rho_{m+1}=\rho_{1}$ and let $\rho_{i}=\mathbb{R}_{\geq 0} \eta_{i}$ for primitive vectors $\eta_{1}, \ldots, \eta_{m}$ in $N$. Choose a basis $n_{1}, n_{2}$ for $N$ with $n_{1}=\eta_{1}$ and write $\eta_{1}=a_{i} n_{1}+b_{i} n_{2}$. Then $N / N^{\prime}=N /\left\langle\eta_{1}, \ldots, \eta_{m}\right\rangle$ is cyclic of order $\operatorname{gcd}\left\{b_{1}, \ldots, b_{m}\right\}$. On the other hand for $(1 \leq i \leq m-1), \mathrm{Cl}\left(U_{\tau_{i}}\right)$ is cyclic of $\operatorname{order}\left|\operatorname{det}\left[\begin{array}{c}a_{i} a_{i+1} \\ b_{i} b_{i+1}\end{array}\right]\right|$ and $\left|\operatorname{Cl}\left(U_{\tau_{m}}\right)\right|=\left|\operatorname{det}\left[\begin{array}{cc}a_{m} & 1 \\ b_{m} & 1\end{array}\right]\right|$ by (2). Since $\operatorname{gcd}\left(a_{i}, b_{i}\right)=1$ for each $i$, an easy calculation shows $\operatorname{gcd}\left\{\left|\operatorname{Cl}\left(U_{\tau_{i}}\right)\right|\right\}_{i=1}^{m}=\operatorname{gcd}\left\{b_{1}, \ldots, b_{m}\right\}$.

To determine the $p$-subgroups of $\mathrm{B}^{\prime}\left(T_{N} / X\right)$ for each prime number $p$, we introduce some additional notation and terminology. Let $\Gamma$ be a finite edgeweighted graph such that to each edge $E$ is associated the positive integer weight $(E)$. Let $v_{p}$ be the $p$-adic valuation on $\mathbb{Z}$ and set the $p$-weight of $E=$ weight $_{p}(E)=v_{p}($ weight $(E))$. If $\Gamma_{1}$ is a subgraph of $\Gamma$, let weight ${ }_{p}\left(\Gamma_{1}\right)=$ $\sum$ weight $_{p}(E)$ where the summation is over all edges $E$ in $\Gamma_{1}$. A p-maximal spanning tree for $\Gamma$ is a spanning tree $T$ for $\Gamma$ such that weight ${ }_{p}(T)$ is maximal among the $p$-weights of all spanning trees. It is clear that every connected graph has a $p$-maximal spanning tree. Let $T$ be a $p$-maximal spanning tree for $\Gamma$ and let $c$ denote an edge of $\Gamma$ which is not an edge of $T$. Since $T$ is a spanning tree, the subgraph $\Gamma_{1}$ of $\Gamma$ obtained by adding the edge $c$ to $T$ contains a cycle $Z$ which is unique since there is a unique path between any two vertices of the tree $T$. Suppose there is some edge $E$ in $Z$ with weight $(E)<$ weight $_{p}(c)$. Then we could obtain a spanning tree of larger $p$-weight by deleting the edge $E$ from $\Gamma_{1}$. This means that if $T$ is a $p$-maximal spanning tree for $\Gamma, c$ is an edge of $\Gamma$ not in $T$ and $Z$ is the unique cycle in the graph $T \cup\{c\}$, then $c$ is an edge of minimal $p$-weight in $Z$.

Let $\Gamma$ be the (connected) graph we have associated to the fan $\Delta$ whose cones all have dimension $\leq 2$. Assign weights to the edges $\tau_{i-} \rho_{i j}$ of $\Gamma$ by setting weight $\left(\tau_{i-} \rho_{i j}\right)=b_{i}=\left|\mathrm{Cl}\left(U_{\tau_{i}}\right)\right|$ (recall $\mathrm{Cl}\left(U_{\tau_{i}}\right)=\mathbb{Z} / b_{i}$ is cyclic from (2)). Let $T$ be a $p$-maximal spanning tree for $\Gamma$. We have labeled the edges of $\Gamma$ not in $T$ as $\tau_{1-} \rho_{12}, \tau_{2}-\rho_{22}, \ldots, \tau_{e-} \rho_{e 2}$. We call the set of 2-dimensional cones $\left\{\tau_{1}, \ldots, \tau_{e}\right\}$ in $\Delta$ a p-minimal set of cones in $\Delta$. If $\Gamma$ is not connected, then we can decompose $\Delta$ as a union of fans $\Delta_{i}$ with $\Delta_{i} \cap \Delta_{j}=\{0\}$ when $i \neq j$ and the graphs $\Gamma_{i}$ associated to $\Delta_{i}$ are connected. We define a $p$-minimal set of cones in $\Delta$ to be the union of $p$-minimal sets of cones in each $\Delta_{i}$.

Theorem 2.10. Let $\Delta$ be a fan on $N_{\mathbb{R}}$ and assume every cone in $\Delta$ has dimension $\leq 2$. Let $\left\{\tau_{1}, \ldots, \tau_{e}\right\}$ be a p-minimal set of cones in $\Delta$ and let $\left|\mathrm{Cl}\left(U_{\tau_{i}}\right)\right|=b_{i}$. If $X=T_{N} \mathrm{emb}(\Delta)$, then $\mathrm{B}^{\prime}\left(T_{N} / X\right)_{p} \cong\left[\bigoplus_{i=1}^{e} \mathbb{Z} / b_{i}\right]_{p}$. This isomorphism is induced by the epimorphism $\psi$ of (3).

Proof. By Proposition 2.5 and the discussion preceeding the theorem, we can assume the graph $\Gamma$ associated to the fan $\Delta$ is connected. Let $T$ be a $p$ maximal spanning tree for $\Gamma$. Continuing the analysis that was begun in 
the proof of Theorem 2.6 we consider the matrix $[Q \mid C]$ defined there. If $\psi: \bigoplus_{i=1}^{m}\left(\mathbb{Z} \rho_{i 1} \oplus \mathbb{Z} \rho_{i 2}\right) \rightarrow \mathrm{B}^{\prime}\left(T_{N} / X\right)$ is the epimorphism given in (3) and $\widehat{\mathbb{Z}}_{p}$ is the $p$-adic integers, we have an epimorphism

$$
\psi_{p}: \bigoplus_{i=1}^{m}\left(\widehat{\mathbb{Z}}_{p} \rho_{i 1} \oplus \widehat{\mathbb{Z}}_{p} \rho_{i 2}\right) \rightarrow \mathrm{B}^{\prime}\left(T_{N} / X\right)_{p} .
$$

It follows from Lemma 2.7 that $\left\{c_{1}, \ldots, c_{e}\right\}$ generates $\operatorname{coker}(\alpha+\beta)$ so $\left\{\psi_{p}\left(c_{1}\right)\right.$, $\left.\ldots, \psi_{p}\left(c_{e}\right)\right\}$ generates $\mathrm{B}^{\prime}\left(T_{N} / X\right)_{p}$. We check

$$
0=\left\langle\psi_{p}\left(c_{j}\right)\right\rangle \cap\left\langle\psi_{p}\left(c_{1}\right), \ldots, \psi_{p}\left(c_{j-1}\right), \psi_{p}\left(c_{j+1}\right), \ldots, \psi_{p}\left(c_{e}\right)\right\rangle
$$

and $\psi_{p}\left(c_{j}\right)$ has order $p^{v_{p}\left(b_{j}\right)}$ for $1 \leq j \leq e$ by identifying these elements in $\mathbf{B}^{\prime}\left(T_{N} / X\right)$ with their corresponding preimages $c_{j}+$ image $(\alpha+\beta) \in \operatorname{coker}(\alpha+\beta)$ in (3) and then checking the corresponding statements in $\operatorname{coker}(\alpha+\beta)$.

Fix $j$ and let $\pi$ be a permutation of $\{1, \ldots, m\}$ with $\pi$ chosen so $\pi(1)=j$ where the edge $c_{j}=\tau_{j-} \rho_{s}$ and the cycle in $T \cup\left\{c_{j}\right\}$ is

$$
\tau_{j}=\tau_{\pi(1)-} \rho_{1-} \tau_{\pi(2)-} \rho_{2-} \cdots-\tau_{\pi(s)-} \rho_{s-} \tau_{\pi(1)} .
$$

Choose the vertex $\tau_{\pi(s)}$ as the root node for $T$. By Lemma 2.7 we know the columns of $[B \mid C]$ form a basis for $\bigoplus_{i=1}^{m}\left(\mathbb{Z} \rho_{i 1} \oplus \mathbb{Z} \rho_{i 2}\right)$. The column space of $B$ is a submodule of image $(\alpha+\beta)$. Project the submodule $\widehat{\mathbb{Z}}_{p} \beta_{1}+\cdots+\widehat{\mathbb{Z}}_{p} \beta_{m}$ of image $(\alpha+\beta)$ onto a $\widehat{\mathbb{Z}}_{p}$-submodule of the column space of $C$ over $\widehat{\mathbb{Z}}_{p}$. Then $\operatorname{coker}(\alpha+\beta)_{p}$ is the quotient module.

If $s+1 \leq i \leq m$ we check the projection of $\beta_{\pi(i)}$ on $\widehat{\mathbb{Z}}_{p} c_{j}$ is 0 . The selection of $\tau_{\pi(s)}$ as the root node for $T$ gives a partial order on the vertices of $T$. Let $T_{i}$ be the subtree of $T$ with root node $\rho_{\pi(i) 2}$. This means the vertices $v$ in $T_{i}$ are those for which the unique path from $v$ to $\tau_{\pi(s)}$ contains $\rho_{\pi(i) 2}$. In the expression for $\beta_{\pi(i)}$ as a linear combination of the columns of $[B \mid C]$, the columns of $C$ that appear are those $c_{k}$ which when considered as edges of $\Gamma$ are incident to some vertex in $T_{i}$ (see the proof of Theorem 2.6). But neither $\tau_{j}$ nor $\rho_{s}$ are in $T_{i}$ since $i \geq s+1$ so the projection of $\beta_{\pi(i)}$ on $c_{j}$ has coefficient $=0$.

If $1 \leq i \leq s$ let the projection of $\beta_{\pi(i)}$ on $\bigoplus_{k=1}^{e} \widehat{\mathbb{Z}}_{p} c_{k}$ be $\sum_{k=1}^{e} d_{k i} c_{k}$. We say in the proof of Theorem 2.6 that $b_{\pi(i)} \mid d_{j i}$ (where $\left.b_{\pi(i)}=\left|\mathrm{Cl}\left(U_{\pi(i)}\right)\right|\right)$. The projections of $\beta_{\pi(1)}, \ldots, \beta_{\pi(m)}$ on $\bigoplus_{k=1}^{e} \widehat{\mathbb{Z}}_{p} c_{k}$ are the columns of the $e \times m$ matrix $\left(d_{k i}\right)$. We have observed above that $d_{j(s+1)}=\cdots=d_{j m}=0$. The definitions of $\beta_{j}$ and $c_{j}$ imply $\beta_{j}=b_{j} c_{j}$ so $d_{k 1}=0$ if $k \neq j$. Also $b_{\pi(i)} \mid d_{j i}$ for $2 \leq i \leq s$ and we chose $j$ with $v_{p}\left(b_{j}\right)=\min \left\{v_{p}\left(b_{\pi(1)}\right), \ldots, v_{p}\left(b_{\pi(s)}\right)\right\}$. Thus after elementary column operations over $\widehat{\mathbb{Z}}_{p}$ the column space of $\left(d_{k i}\right)$ is equal to the column space of

$$
\left[\begin{array}{cccc}
0 & d_{12} & \cdots & d_{1 m} \\
\vdots & \vdots & & \vdots \\
0 & d_{(j-1) 2} & & \\
b_{j} & 0 & \cdots & 0 \\
0 & d_{(j+1) 2} & & \\
\vdots & \vdots & & \vdots \\
0 & d_{e 2} & \cdots & d_{e m}
\end{array}\right]
$$


Therefore $c_{1}, \ldots, c_{e}$ represents a basis for $\operatorname{coker}(\alpha+\beta)_{p}, \quad \mathbf{B}^{\prime}\left(T_{N} / X\right)_{p}=$ $\left\langle\psi_{p}\left(c_{1}\right)\right\rangle \oplus \cdots \oplus\left\langle\psi_{p}\left(c_{e}\right)\right\rangle$, and $\left\langle\psi_{p}\left(c_{j}\right)\right\rangle$ is cyclic of order $p^{v_{p}\left(b_{j}\right)}$.

\section{ACKNOWLEDGMENT}

This article was written while the first author visited at Florida Atlantic University. He thanks department chairman Jim Brewer for his hospitality.

\section{REFERENCES}

1. N. Biggs, Algebraic graph theory, Cambridge Univ. Press, London, 1974.

2. E. Brieskorn, Rational singularitäten komplexer Flächen, Invent. Math. 4 (1968), 336-358.

3. T. Ford, On the Brauer group of $k\left[x_{1}, \ldots, x_{n}, 1 / f\right]$, J. Algebra 122 (1989), 410-424.

4. __ On the Brauer group of a localization, J. Algebra 147 (1992), 365-378.

5. R. Fossum, The divisor class group of a Krull domain, Springer-Verlag, New York, 1973.

6. J. Gamst and K. Hoechsmann, Quaternions generalises, C. R. Acad. Sci. Paris 269A (1969), $560-562$.

7. A Grothendieck, Le groupe de Brauer. II, Dix Exposés sur la Cohomologie des Schémas, North-Holland, Amsterdam, 1968.

8. R. Hoobler, Functors of graded rings, Methods in Ring Theory ( $F$. van Oystaeyen, ed.), NATO ASI Series, Reidel, Dordrecht, 1984, pp. 161-170.

9. __ When is $\operatorname{Br}(X)=\operatorname{Br}^{\prime}(X)$ ?, Brauer Groups in Ring Theory and Algebraic Geometry, Lecture Notes in Math., Vol. 917, Springer-Verlag, New York/Berlin, 1982, pp. 231-245.

10. A Magid, Brauer groups of linear algebraic groups with characters, Proc. Amer. Math. Soc. 71 (1978), 164-168.

11. J. Milne, Etale cohomology, Princeton Univ. Press, Princeton, N. J., 1980.

12. T. Oda, Convex bodies and algebraic geometry, Springer-Verlag, Berlin and Heidelberg, 1988.

13. M. Raynaud, Anneaux locaux Henséliens, Lecture Notes in Math., Vol. 169, SpringerVerlag, Berlin, 1970.

14. O. F. G. Schilling, The theory of valuations, Math. Surveys, No. 4, Amer. Math. Soc., Providence, R. I., 1950.

15. R. Strano, On the étale cohomology of Hensel rings, Comm. Algebra 12 (1984), 2195-2211.

Department of Mathematics, Colorado State University, Fort Collins, Colorado 80523

E-mail address: demeyer@euclid.math.colostate.edu

Department of Mathematics, Florida Atlantic University, Boca Raton, Florida 33431

E-mail address: ford@acc.fau.edu 